The University of Southern Mississippi

The Aquila Digital Community

Faculty Publications

$1-28-2019$

\title{
A Learning-Based Automatic Segmentation and Quantification Method on Left Ventricle in Gated Myocardial Perfusion SPECT Imaging: A Feasibility Study
}

\author{
Tonghe Wang \\ Emory University \\ Yang Lei \\ Emory University \\ Haipeng Tang \\ University of Southern Mississippi \\ Zhou He \\ University of Southern Mississippi \\ Richard Castillo \\ Emory University
}

Perforexthpagrefordaftlitianaloakthar:Shttps://aquila.usm.edu/fac_pubs

Part of the Analytical, Diagnostic and Therapeutic Techniques and Equipment Commons

\section{Recommended Citation}

Wang, T., Lei, Y., Tang, H., He, Z., Castillo, R., Wang, C., Li, D., Higgins, K., Liu, T., Curran, W. J., Curran, W. J., Zhou, W., Yang, X. (2019). A Learning-Based Automatic Segmentation and Quantification Method on Left Ventricle in Gated Myocardial Perfusion SPECT Imaging: A Feasibility Study. Journal of Nuclear Cardiology, 1-12.

Available at: https://aquila.usm.edu/fac_pubs/15833

This Article is brought to you for free and open access by The Aquila Digital Community. It has been accepted for inclusion in Faculty Publications by an authorized administrator of The Aquila Digital Community. For more information, please contact Joshua.Cromwell@usm.edu. 


\section{Authors}

Tonghe Wang, Yang Lei, Haipeng Tang, Zhou He, Richard Castillo, Cheng Wang, Dianfu Li, Kristin Higgins, Tian Liu, Walter J. Curran, Walter J. Curran, Weihua Zhou, and Xiaofeng Yang 


\title{
A Learning-based Automatic Segmentation and Quantification Method on Left Ventricle in Gated Myocardial Perfusion SPECT Imaging: A Feasibility
}

\author{
Study

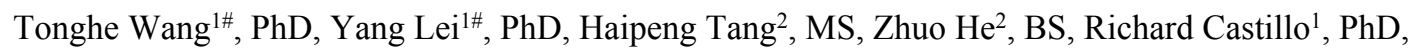 \\ Cheng Wang ${ }^{3}$, MD, Dianfu Li³ ${ }^{3}$ MD , Kristin Higgins ${ }^{1}$, MD, Tian Liu ${ }^{1}, \mathrm{PhD}$, \\ Walter J. Curran ${ }^{1}, \mathrm{MD}$, Weihua Zhou ${ }^{2 *}, \mathrm{PhD}$, and Xiaofeng Yang ${ }^{1 *}, \mathrm{PhD}$ \\ ${ }^{1}$ Department of Radiation Oncology and Winship Cancer Institute, Emory University, Atlanta, GA 30322 \\ ${ }^{2}$ School of Computing, University of Southern Mississippi, Long Beach, MS 39560 \\ ${ }^{3}$ Department of Cardiology, The First Affiliated Hospital of Nanjing Medical University, Nanjing, Jiangsu, China \\ \# Equal contribution, co-first author
}

Financial support: This research is supported in part by the National Cancer Institute of the National Institutes of Health under award number R01CA215718 and Emory Winship Cancer Institute pilot grant. This research is also supported by the American Heart Association under Award number 17AIREA33700016. 


\title{
A Learning-based Automatic Segmentation and Quantification Method on Left Ventricle in Gated Myocardial Perfusion SPECT Imaging: A Feasibility \\ Study
}

\begin{abstract}
Background: The performance of left ventricular (LV) functional assessment using gated myocardial perfusion SPECT (MPS) relies on the accuracy of segmentation. Current methods require manual adjustments that are tedious and subjective. We propose a novel machine-learning-based method to automatically segment LV myocardium and measure its volume in gated MPS imaging without human intervention. Methods: We used an end-to-end fully convolutional neural network to segment LV myocardium by delineating its endocardial and epicardial surface. A novel compound loss function, which encourages similarity and penalizes discrepancy between prediction and training dataset, is utilized in training stage to achieve excellent performance. We retrospectively investigated 32 normal patients and 24 abnormal patients, whose LV myocardial contours automatically segmented by our method were compared with those delineated by physicians as the ground truth. Results: The results of our method demonstrated very good agreement with the ground truth. The average DSC metrics and Hausdorff distance of the contours delineated by our method are larger than 0.900 and less than $1 \mathrm{~cm}$, respectively, among all $32+24$ patients of all phases. The correlation coefficient of the LV myocardium volume between ground truth and our results is $0.910 \pm 0.061(\mathrm{P}<0.001)$, and the mean relative error of LV myocardium volume is $-1.09 \pm 3.66 \%$. Conclusion: These results strongly indicate the feasibility of our method in accurately quantifing LV myocardium volume change over the cardiac cycle. The learning-based segmentation method in gated MPS imaging has great promise for clinical use.
\end{abstract}

Keywords: myocardial perfusion, SPECT, segmentation, machine learning 
Abbreviations: SPECT: single-photon emission computed tomography; MPS: myocardial perfusion SPECT; LV: left ventricular; CT: computed tomography; MRI: magnetic resonance imaging; BCE: binary cross entropy; DSC: Dice similarity coefficient; EF: ejection fraction; EDV: end-diastolic volume; ESV: end-systolic volume. 


\section{INTRODUCTION}

Myocardial perfusion SPECT (MPS) has been one of the most important imaging modalities for the assessment of left ventricular (LV) function (1-4). Photons emitted by an injected radioactive perfusion tracer taken up by the LV myocardium are detected to reconstruct perfusion images. With electrocardiographic gating, MPS provides 8 or 16 volumetric perfusion image sets corresponding to different phases of the cardiac cycle (5). Evaluations are performed based on these images by visual and quantitative estimation of the variation of LV during the cardiac cycle (6). LV contractile functional indices can then be derived from MPS images for the diagnosis/prognosis of coronary artery disease and patient risk assessment (7-12).

The fidelity of LV function assessment by MPS directly relates to the quantification accuracy of the LV myocardium volume $(13,14)$. The measurement of LV myocardium volume starts with delineation of epicardial and endocardial boundaries on the perfusion images, and is calculated as the volume bounded by the epicardial and endocardial surface. Manual segmentation is tedious when it involves studies on multiple volumetric phase images of the cardiac cycle, and is dependent on observers' experience. It is desirable to develop an observerindependent segmentation method to improve efficiency and reproducibility with comparable accuracy.

Current automated methods extract the epicardial and endocardial boundaries based on general assumptions and rules with empirical parameters. For example, commercially available methods estimate the profiles of the myocardium by identifying the maximal myocardial count, then applying Gaussian fitting with empirical standard deviation or threshold to extract endocardial and epicardial boundaries $(15,16)$. This method is easy and fast to implement, though it neglects the anatomical variations and pathology abnormalities among different patients. Studies have shown that LV myocardium volume would be over- or underestimated by this method $(17,18)$, and manual adjustments are usually required (14).

In recent years, machine learning methods are being integrated into segmentation studies. They have been shown to feature better results while requiring less time than traditional methods for CT and MR images (19-21) due to its data-driven approaches toward automatically learning image features and model parameters. Compared 
with these common imaging modalities, MPS images have advantages for machine learning methods in that image size is greatly reduced and with higher image contrast, which leads to more efficient extraction of global features from the whole image set during the training stage. Thus, the machine learning method is promising in automatic MPS image segmentation.

In this paper, we propose a novel machine-learning based method to automatically segment LV myocardium by delineating its endocardial and epicardial surface, and measure its volume in gated MPS imaging. Our method uses a multi-class 3D V-Net, which is an end-to-end fully convolutional neural network. A compound loss function, which simultaneously encourages similarity and penalizes discrepancy between prediction and training datasets, was utilized in training stage to achieve excellent performance. To evaluate our proposed method, we retrospectively investigated 32 normal patients and 24 abnormal patients with clinicallyacquired MPS. The LV myocardium was segmented by our proposed method and compared with ground truth approved by physicians for evaluation on a total of $32+\underline{24 \text { patients. }}$

\section{METHODS AND MATERIALS}

The proposed SPECT LV myocardium segmentation method consists of a training stage and a segmentation stage. For a given SPECT image dataset, the clinically-implemented physician-drawn contours of the endocardial and epicardial surface of myocardium are available. These clinical contours were used as the learning-based target of the SPECT image. The region within endocardial surfaces, region within epicardial surfaces, and background region are regarded as training and segmenting classes in our method. The original SPECT images were first automatically cropped into $32 \times 32 \times 16$ voxels to reduce background region: a threshold was used to get rid of background and the centroid of the active heart region was then calculated, based on which a 32x32x16 voxel region was cropped to cover the active heart region. A volume-based deep learning network was trained based on such extracted SPECT image volume. The 3D multi-class V-Net architecture was used to enable voxel-wise error back-propagation during the training stage, and directly outputting an equal-sized prediction patch with the input patch during the testing procedure (22). By up-scaling low [4x4x2], modest [8x8x4], and high-level [16x16x8] feature volumes at each forwarding path from left to right portions of the 
hidden network using additional deconvolutional layers, and incorporating last output feature volume, the softmax function was employed on these equal-sized feature volumes to obtain final contour prediction. The Adam gradient decent optimizer was employed to train the V-Net. We used the whole volume as a patch and the batch size (number of patches) is 20. The number of epochs is 180 . Compound loss supervision was then integrated into this prediction by considering both binary cross entropy and Dice loss to supervise the back-propagation of gradients for parameter updating in each training epoch. During the segmentation stage, the new arrival 3D patient

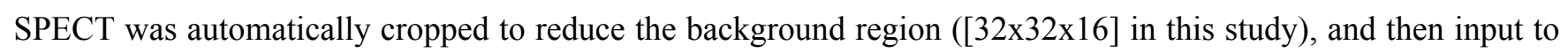
the trained networks. The output volume was a multi-class contour probability maps. Finally, the segmentation was generated by thresholding the probability maps larger than 0.5 . Fig. 1 outlines the workflow schematic of our segmentation method.

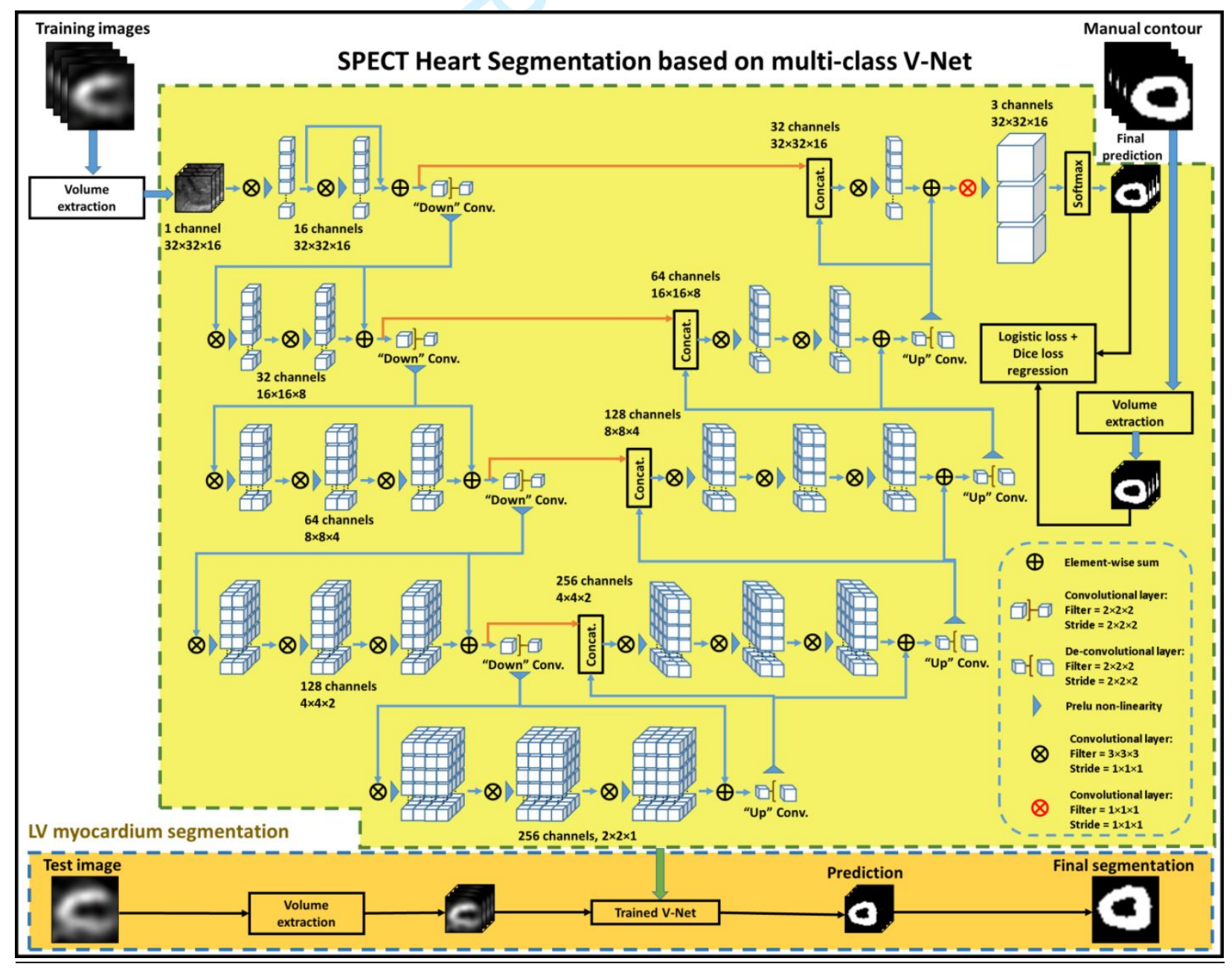


Fig. 1 Schematic flow chart of the proposed algorithm for LV segmentation. The upper part of this figure shows the training stage of our proposed method. The upper part also shows the V-Net architecture which has single channel volume input and 3 channels (background, region within endocardium, and region within epicardium) volume output. The lower part (brown) shows the segmentation stage. In segmentation stage, a new SPECT heart image is fed into the well-trained model to get the segmentation.

In this study, we propose a compound loss function incorporating both the effectiveness of logistic loss and Dice loss functions to supervise our network. Since the optimization of the prediction task is a binary regression, we first used the voxel-wise binary cross entropy (BCE) loss as the logistic loss function. The $\mathrm{BCE}$ loss is defined as follows:

$$
L_{B C E}(C, \hat{C})=-\sum_{j} C_{j} \log \hat{C}_{j}+\left(1-C_{j}\right) \log \left(1-\hat{C}_{j}\right),
$$

where $C_{j}$ and $\hat{C}_{j}$ denote the $j$ th voxel in clinical contour $C$ and prediction $\hat{C}$, respectively.

The endocardial surface contour often occupies a small region of the MPS images as compared with epicardial surface contour. This may cause the network to ignore segmented regions and bias network output towards the background. The learning process can be trapped in local minima and unable to obtain accurate results. To address this issue, we additionally incorporated the logistic loss with Dice loss in the final stage as the final objective function. The Dice loss for segmentation was originally proposed in a 3D model defined as:

$$
L_{D i c e}(C, \hat{C})=1-\frac{2 \times V(C \cap \hat{C})}{V(C)+V(\hat{C})}
$$

where $V$ indicates the volume of the region enclosed in the contours.

The compound loss function is a combination of the above BCE and Dice loss functions, which are related to the dissimilarity and similarity between prediction and training dataset, respectively. It is defined as follows:

$$
L_{\text {final }}(C, \hat{C})=L_{B C E}(C, \hat{C})+\mu L_{\text {Dice }}(C, \hat{C}),
$$

where $\mu$ is an empirical parameter, which balances the loss from binary cross entropy and Dice coefficient. In order to fairly compare the performance of the method on different patients, our hyper parameters of the network were fixed before we conducted the leave-one-out experiments. The batch size was set to 20 . The number of epochs was set to 180 . For the parameter $\mu$, we employed 4-fold cross validation to evaluate its setting. It was shown that the performance is not sensitive when $\mu$ is between $[0.7,1.3]$, thus we set $\mu=1$. 
To evaluate the performance of the proposed method for LV myocardium segmentation, we compared the difference of contours generated by our method with clinical contours. In this retrospective study, we studied the dataset of 32 patients without hypertension, diabetes, heart dysfunction, family history of heart diseases (mean \pm STD age: $63 \pm 10,23$ males, 9 females). The cohort of 32 patients were used to evaluate our method using the leave-one-out cross-validation. For one test patient, the model is trained by the rest 31 patients. The model is initialized and re-trained for next test patient by another group of 31 patients. The training dataset and testing dataset are separated and independent during each study. In addition, 24 patients (mean \pm STD age: $57 \pm 10,17$ males, 7 females) diagnosed with myocardial ischemia ranging from mild, moderate to severe extents were also included to further test the proposed segmentation method with a leave-one-out validation strategy. Institutional review board approval was obtained with no informed consent required for this HIPAA-compliant retrospective analysis. Each patient underwent 8-frame ECG-gated resting SPECT performed 30 minutes after injection of 20$30 \mathrm{mCi}$ Tc-99m sestamibi. The SPECT images were acquired by a dual-headed camera (CardioMD, Philips Medical Systems) using a standard resting protocol. The acquisition parameters were $20 \%$ energy window around $140 \mathrm{keV}, 180^{\circ}$ orbit, 32 steps with 25 seconds per step, 8-bin gating and 64 projections per gate. Images were reconstructed into transaxial slices by ordered subsets expectation maximization with 3 iterations and 10 subsets, with Butterworth filter of power 10 and cutoff frequency of 0.3 cycles $/ \mathrm{cm}$. The reconstructed voxel dimensions of each SPECT image volume was $6.4 \times 6.4 \times 6.4 \mathrm{~mm}^{3}$.

Each patient had contours of myocardium with endocardial and epicardial surface delineated and approved by physicians, which were treated as the ground truth. Corresponding contours were also generated by our proposed method. The volume between endocardial and epicardial surface was considered as myocardium where the LV myocardium volume is calculated. We first visually checked the similarity of the contours between ground truth and the results of the proposed method. Quantitatively, we characterized the accuracy of the proposed method by calculating two widely-used metrics: Dice similarity coefficient (DSC) and Hausdorff distance. The DSC describes the overlapping of the segmented volumes between ground truth and proposed method, which can be calculated by 1-Eq. (2), with $C$ and $\hat{C}$ are the contours of ground truth and proposed method 
results, respectively. A magnitude of DSC closer to 1 indicates higher overlapping with ground truth, thus high accuracy of the proposed method. The DSC metrics were calculated on the contours of endocardial surface and epicardial surface individually, as well as the combined myocardium contour. Hausdorff distance measures the difference of two contours in distance. It is defined as the maximum of the closest distances from each point on one contour to all points on the other contour, (23) i.e.,

$$
H(C, \hat{C})=\max \left\{\max _{a \in C} \min _{b \in \hat{C}}\|a-b\|, \max _{b \in \hat{C}} \min _{a \in C}\|a-b\|\right\}
$$

where $a$ and $b$ are points on contours of ground truth $C$ and proposed method results $\hat{C}$, respectively. $\|a-b\|$ represents the Euclidean distance between point $a$ and $b$. A smaller Hausdorff distance means higher similarity between the two contours. Hausdorff distance metrics were calculated on the contours of endocardial surface and epicardial surface individually. To determine interobserver variability, the DSC and Hausdorff distance metrics were also calculated on the contours delineated by a second observer for three randomly selected patients with all phases from the total dataset.

The accuracy of the LV myocardium volume was evaluated by Pearson Correlation analysis between ground truth and the results of proposed method among all patients for each gating phase $(0,1 / 8, \ldots 7 / 8)$, and its correlation coefficient $(r)$ and $\mathrm{P}$ value were calculated. A correlation coefficient closer to 1 indicates higher accuracy of proposed method, and a $\mathrm{P}$ value of less than 0.05 was considered to be statistically significant. The relative error of measured LV myocardium volume was calculated as the difference between ground truth and the results of proposed method relative to ground truth. A Bland-Altman figure was then plotted to show the absolute systematic bias in error and dependence of error on volume size.

Moreover, we calculated LV ejection fraction (EF) to further evaluate our method. We considered the region within endocardial surface of myocardium as the LV cavity. The maximum and minimum volumes within endocardial surface among all phases were then used as end-diastolic volume (EDV) and end-systolic volume $(\mathrm{ESV})$ respectively to calculate $\mathrm{EF}=(\mathrm{EDV}-\mathrm{ESV}) / \mathrm{EDV}$. The EF calculation based on the segmentations of our 
proposed method was compared with that based on the ground truth, as well as compared with results from a commercial software package (Emory Cardiac Toolbox 4.0,Atlanta, USA).

\section{RESULTS}

\section{$\underline{\text { Normal group }}$}

In Fig. 2, the segmentations by the proposed method are compared side-by-side with the clinical ground truth at different slices of gating phase 0 from patient \#1 (normal) as an example. In this case, the LV myocardium volume measured by the proposed method was $191.5 \mathrm{cc}$, underestimated $1.74 \%$ from ground truth $194.9 \mathrm{cc}$.

Fig. 3 shows a side-by-side comparison between the segmentations by the proposed method and ground truth from the same patient as Fig. 2, but across different gating phases of the same slice. The mean and standard deviation (STD) of DSC of endocardial and epicardial surface, and myocardium, and Hausdorff distance of endocardial and epicardial surface among all 32 normal patients are plotted in Fig. 4 for each phase and summarized in Table 1. The average DSC metrics and Hausdorff distance are all larger than 0.900 and less than $1 \mathrm{~cm}$, respectively. The minimum DSC on myocardium is 0.783 which is the only case with DCS less than 0.800 . These results quantitatively demonstrate high accuracy of contours delineated by the proposed method. No case shows unreasonable result among the 32 patients and 8 phases using our method.

The LV myocardium volumes changing with phases are demonstrated in Fig. 5 for patients 01 to 04 . Our method accurately quantified the variation of LV myocardium volume during the cardiac cycle. Note that we present the results of four patients in figures as examples, but similar results can be seen on the other patients. 
Fig. 2 The axial views of patient \#1 (normal) at different slices of gating phase 0 with segmentations of ground truth and proposed method. The black lines indicate the contours of endocardial and epicardial surface.

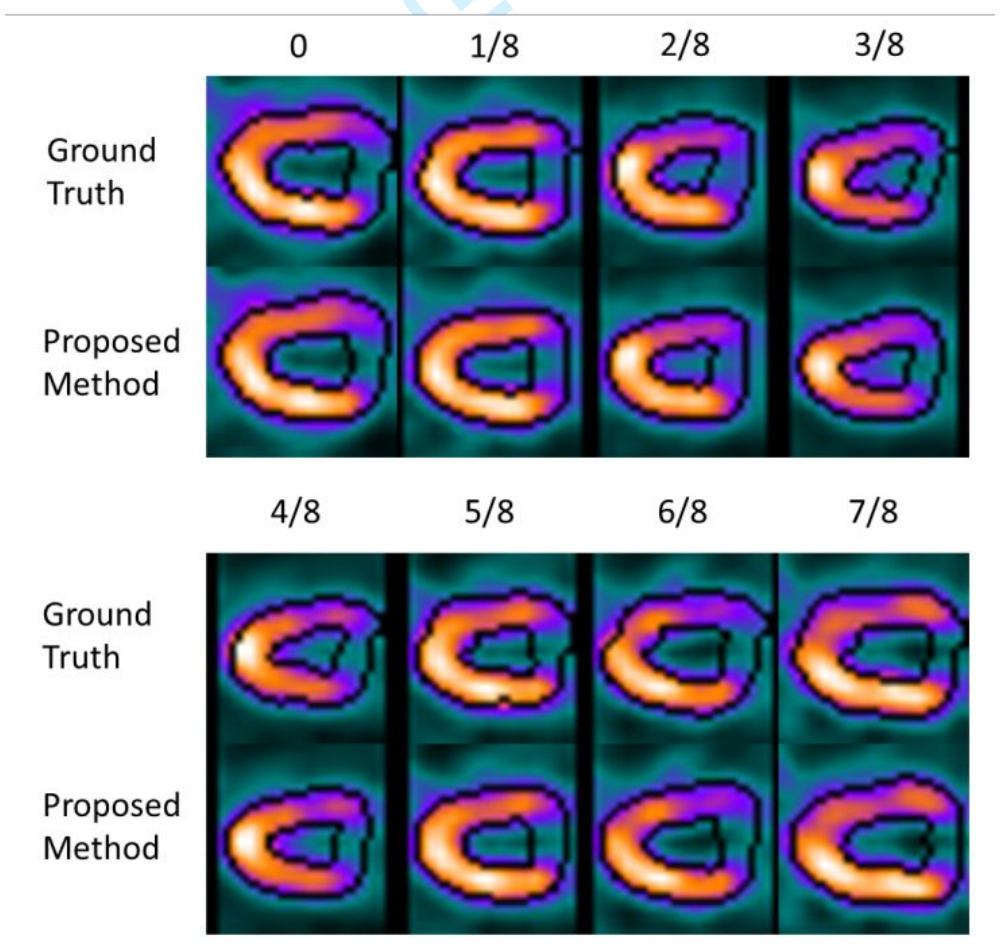

Fig. 3 The axial views of patient \#1 (normal) from phase 0 to $7 / 8$ at same slice with segmentations of ground truth and proposed method. The black lines indicate the contours of endocardial and epicardial surface. 

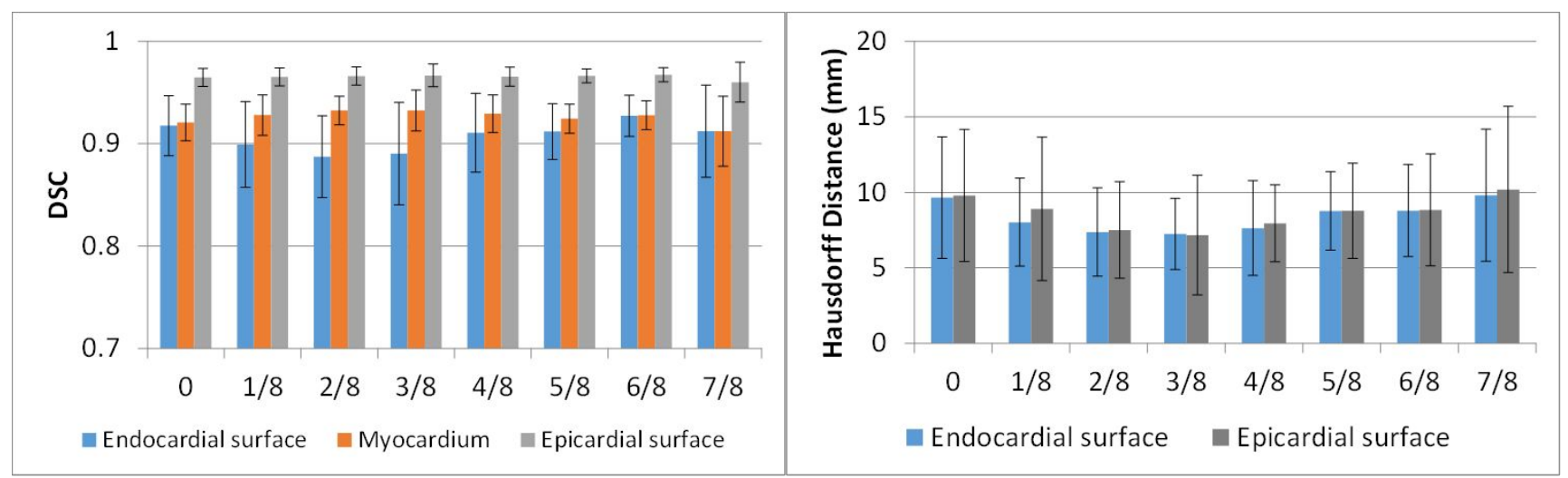

Fig. 4 Mean and STD of DSC and Hausdorff distance of contours between ground truth and proposed method among all 32 normal patients for each phase.

Table 1. Mean \pm STD of DSC and Hausdorff distance among all 32 normal patients.

\begin{tabular}{cccc}
\hline \hline Metrics & Endocardium & Myocardium & Epicardium \\
\hline DSC & $0.907 \pm 0.039$ & $0.926 \pm 0.021$ & $0.965 \pm 0.011$ \\
Hausdorff Distance $(\mathrm{mm})$ & $8.402 \pm 3.317$ & N/A & $8.631 \pm 4.057$ \\
\hline \hline
\end{tabular}

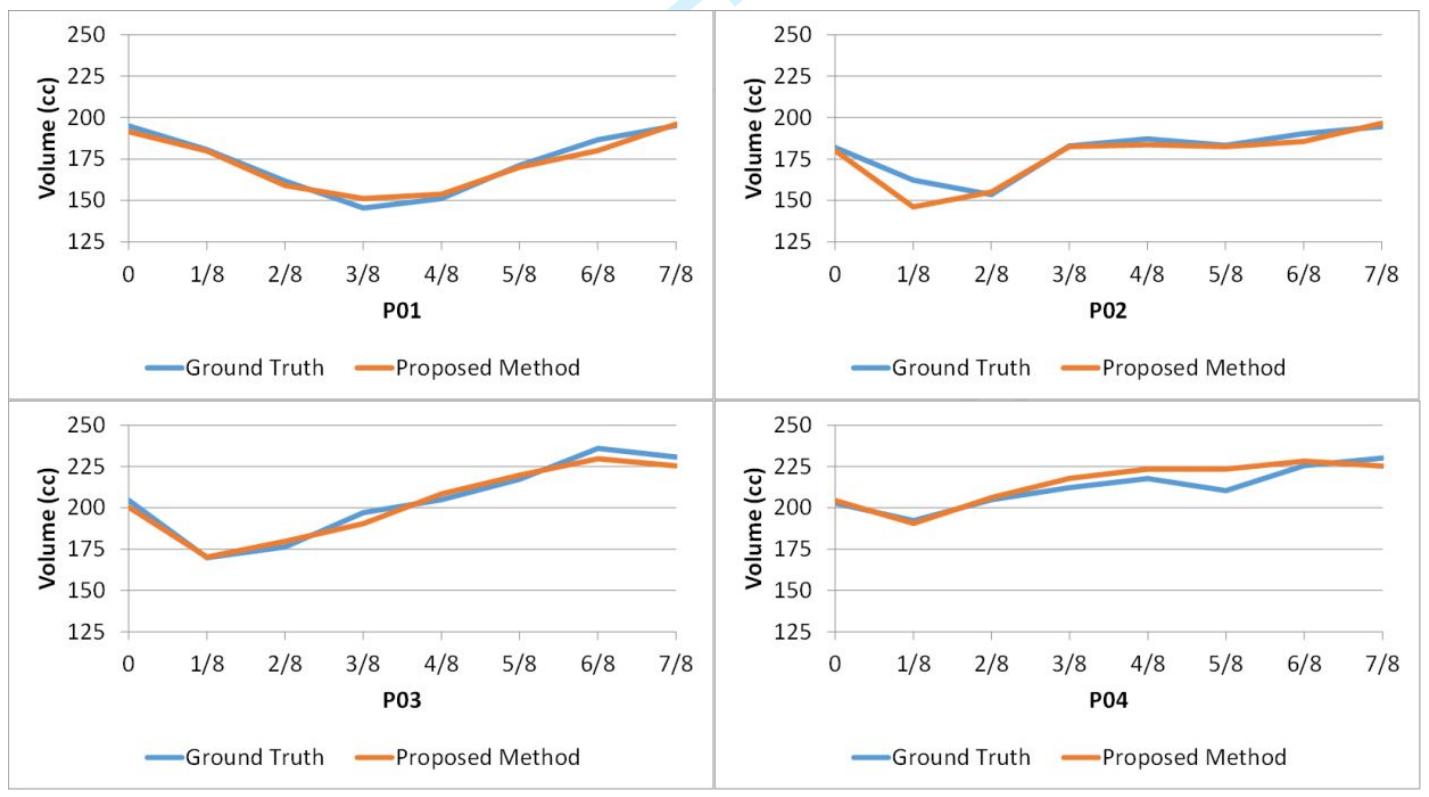

Fig. 5 LV myocardium volumes of ground truth and measured by ground truth at different phases of patient 01 to 04 (normal patients). 
The correlation analysis of LV myocardium volume between ground truth and the proposed method is shown in Fig. 6. Mean (and STD) of $\mathrm{r}$ among all phases is $0.910 \pm 0.061$, and all $\mathrm{P}$ values are less than 0.001 , which indicates statistically significant linear correlation between LV myocardium volumes measured by proposed method and ground truth. The relative error in LV myocardium volume measurement for each phase is presented in Fig. 7 as a Bland-Altman plot. The mean (and STD) relative error among all patients and all phases is $-1.09 \pm 3.66 \%$. The average linear correlation coefficient between volume error and volume size for all phases is $0.222(\mathrm{P}=0.238)$
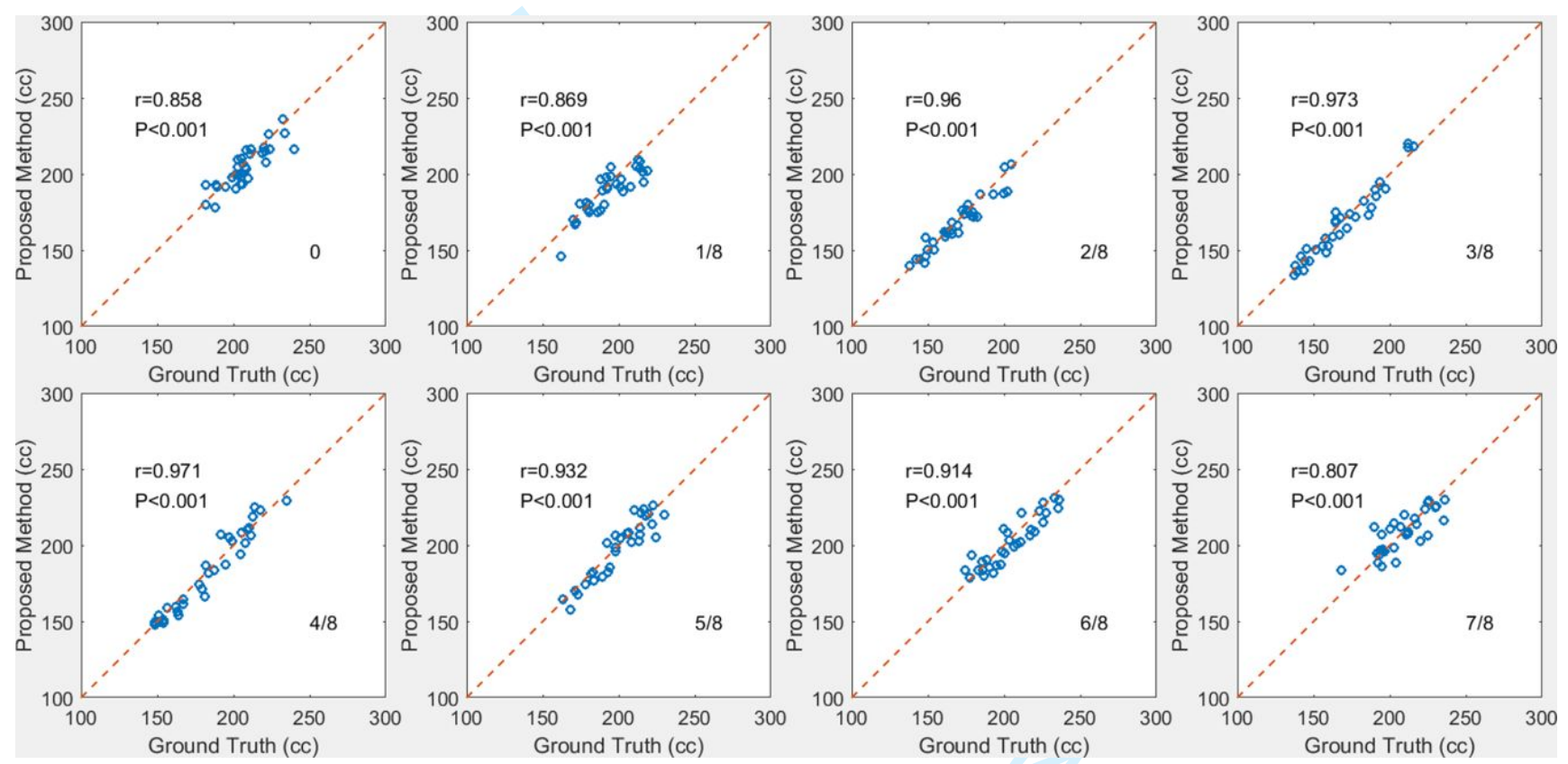

Fig. 6 Correlation analysis of LV myocardium volume between ground truth and proposed method at each gating phase among all 32 normal patients. Blue circle indicates measurement of each patient at that phase, and dashed red line is line of identity. 

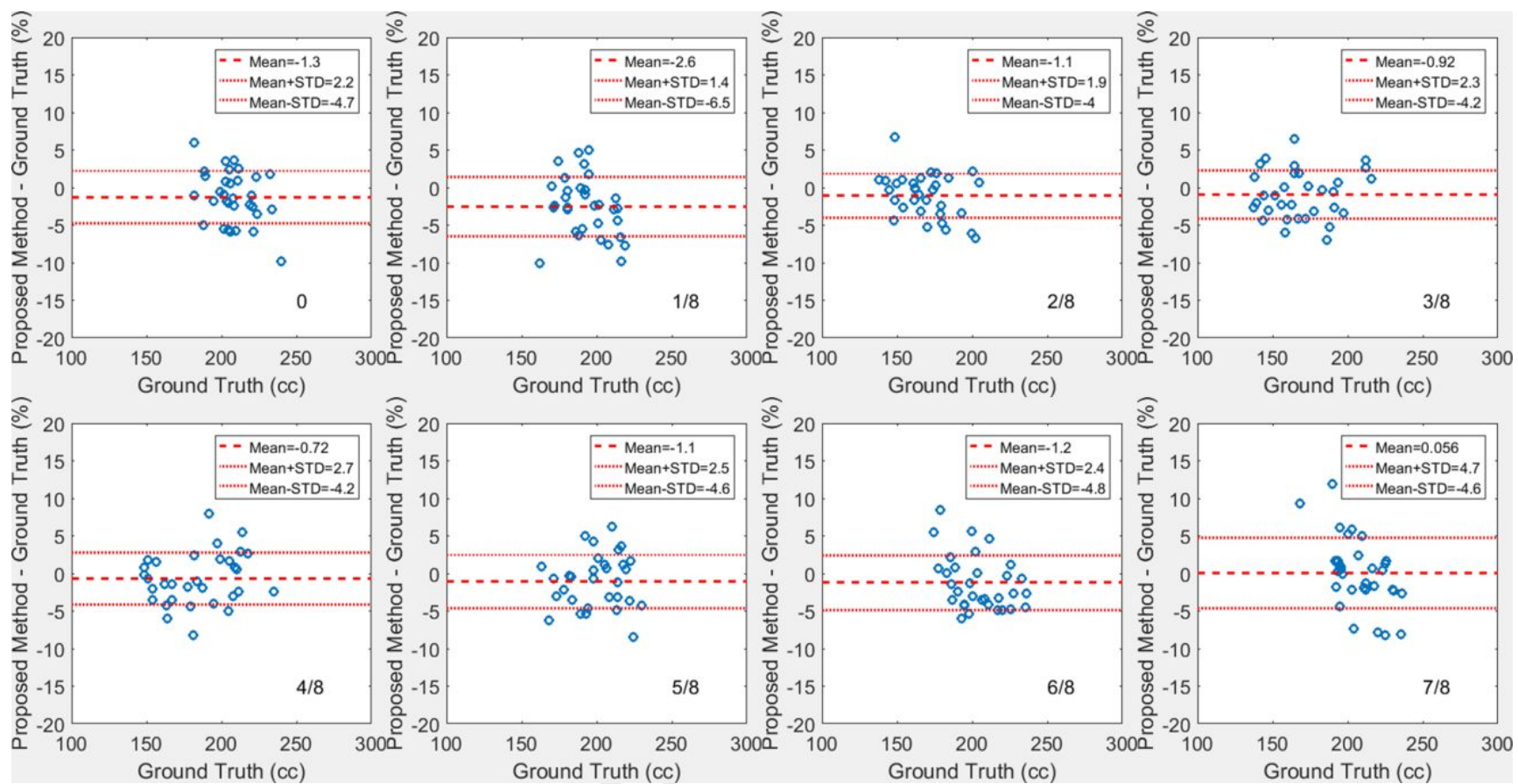

Fig. 7 Relative error of LV myocardium volume measured by the proposed method of each normal patient at each phase.

The EF results on normal patients are calculated based on our results and ground truth, and obtained from commercial software are shown in Fig. 8 as Bland-Altman plots. Good correlation on EF is shown between our results and ground truth $(\mathrm{r}=0.893, \mathrm{P}<0.001)$. Correlation between our results and commercial software is fair ( $\mathrm{r}$ $=0.644, \mathrm{P}<0.001)$. Similar studies on ESV and EDV are shown in Fig. 9. Excellent correlations are found between our method and ground truth for both ESV and EDV, and between our method and commercial software for EDV. The correlation of ESV between our method and commercial software is fair. 

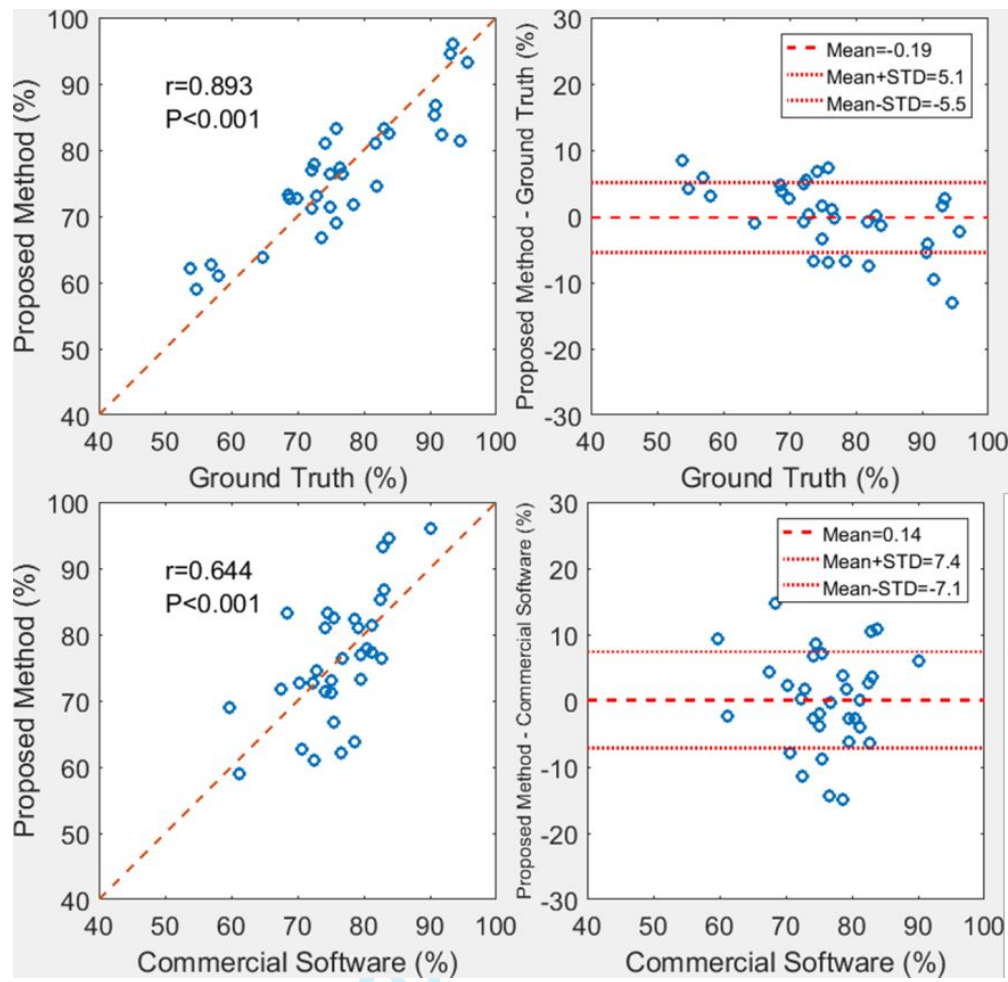

Fig. 8 Left: Correlation analysis of EF between ground truth and proposed method (upper) and between commercial software and proposed method (bottom). Right: Difference of EF between ground truth and proposed method (upper) and between commercial software and proposed method (bottom). 

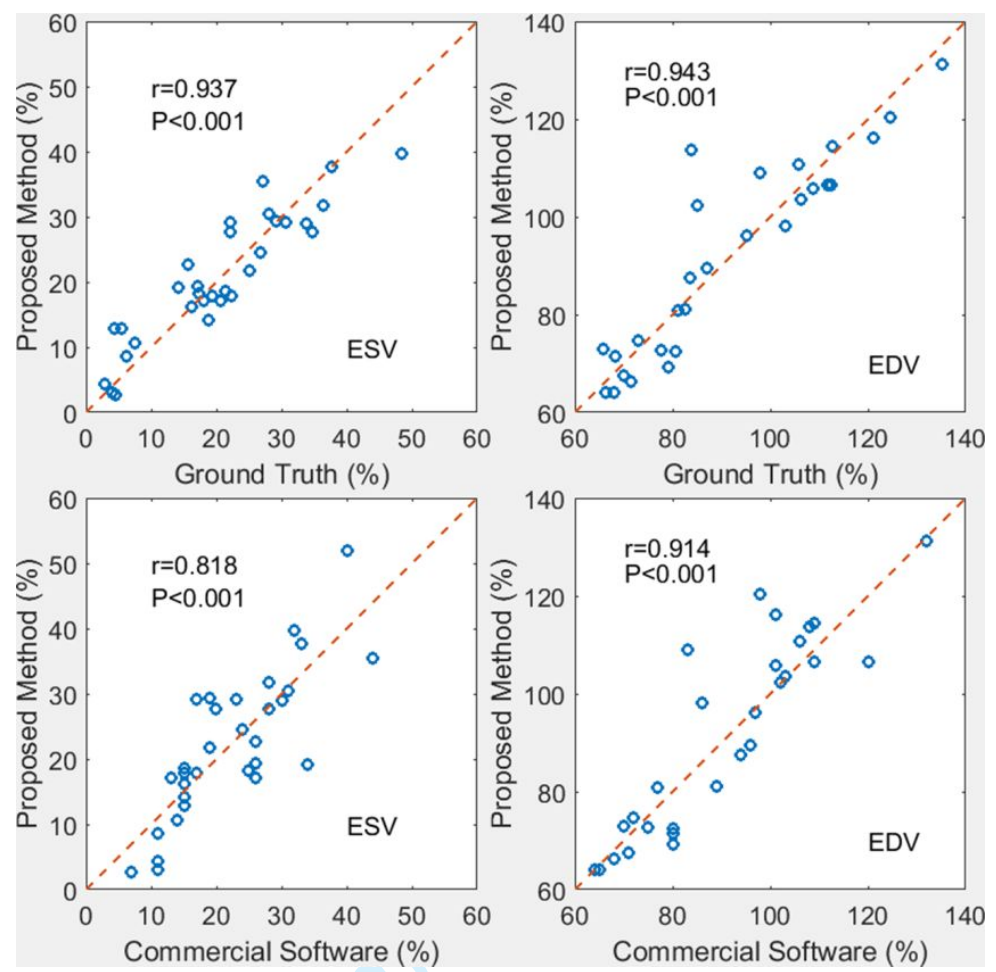

Fig. 9 Correlation analysis of ESV (left) and EDV (right) between ground truth and proposed method (upper) and between commercial software and proposed method (bottom).

\section{Abnormal group}

Fig. 10 demonstrates a side-by-side comparison between our results and the clinical ground truth at different slices of gating phase 0 from patient \#25 (diagnosed with moderate ischemia) as an example. In this case, the LV myocardium volume measured by the proposed method was $212.0 \mathrm{cc}$, overestimated $1.49 \%$ from ground truth $208.9 \mathrm{cc}$. The mean and standard deviation (STD) of DSC of endocardial and epicardial surface, and myocardium, and Hausdorff distance of endocardial and epicardial surface among all 24 abnormal patients are plotted in Fig. 11 for each phase and summarized in Table 2. Overall, the results on abnormal patients are very similar with those of normal patients, with mean DSC larger than 0.9 and Hausdorff distance less than $1 \mathrm{~cm}$. The correlation coefficient of the LV myocardium volume between ground truth and our results is $0.939 \pm 0.103$ $(\mathrm{P}<0.001)$, and the mean relative error of LV myocardium volume is $-0.567 \pm 3.47 \%$. 
Fig. 10 The axial views of patient \#25 (abnormal) at different slices of gating phase 0 with segmentations of ground truth and proposed method. The black lines indicate the contours of endocardial and epicardial surface.
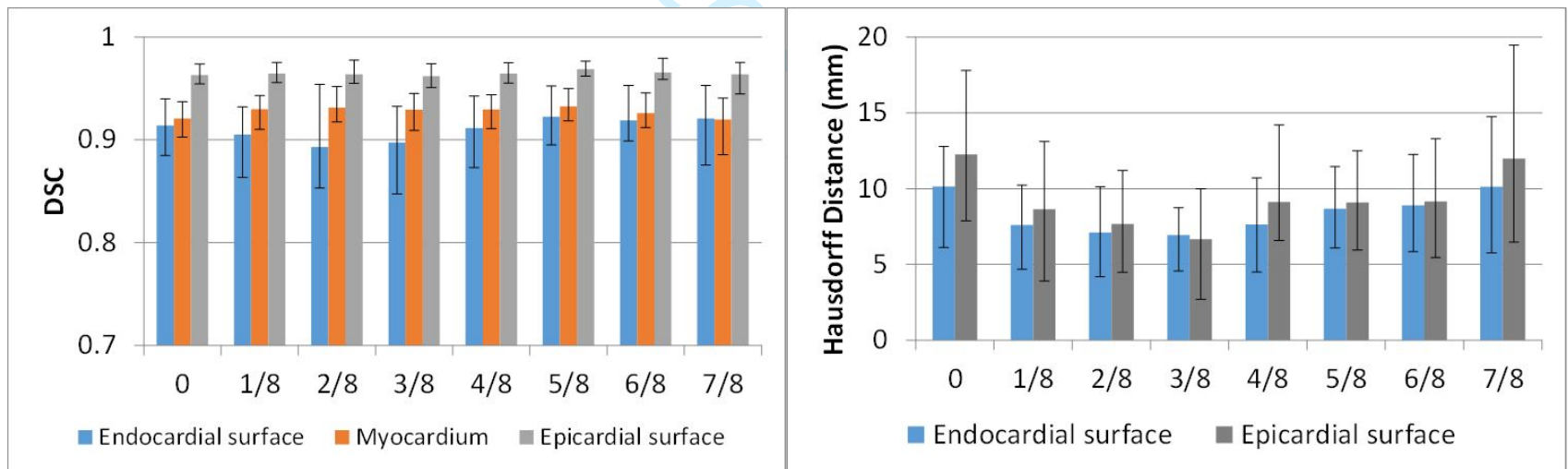

Fig. 11 Mean and STD of DSC and Hausdorff distance of contours between ground truth and proposed method among all 24 abnormal patients for each phase.

Table 2. Mean \pm STD of DSC and Hausdorff distance among all 24 abnormal patients.

\begin{tabular}{cccc}
\hline \hline Metrics & Endocardium & Myocardium & Epicardium \\
\hline DSC & $0.910 \pm 0.037$ & $0.927 \pm 0.018$ & $0.965 \pm 0.011$ \\
Hausdorff Distance $(\mathrm{mm})$ & $8.384 \pm 3.240$ & $\mathrm{~N} / \mathrm{A}$ & $9.310 \pm 5.034$ \\
\hline \hline
\end{tabular}




\section{Interobserver study}

The interobserver study results on normal patients are shown in Fig. 12. The average DSC and Hausdorff distance between contours from two observers are 0.890 and $10.99 \mathrm{~mm}$, respectively, which is higher but still comparable to the discrepancy of our method.

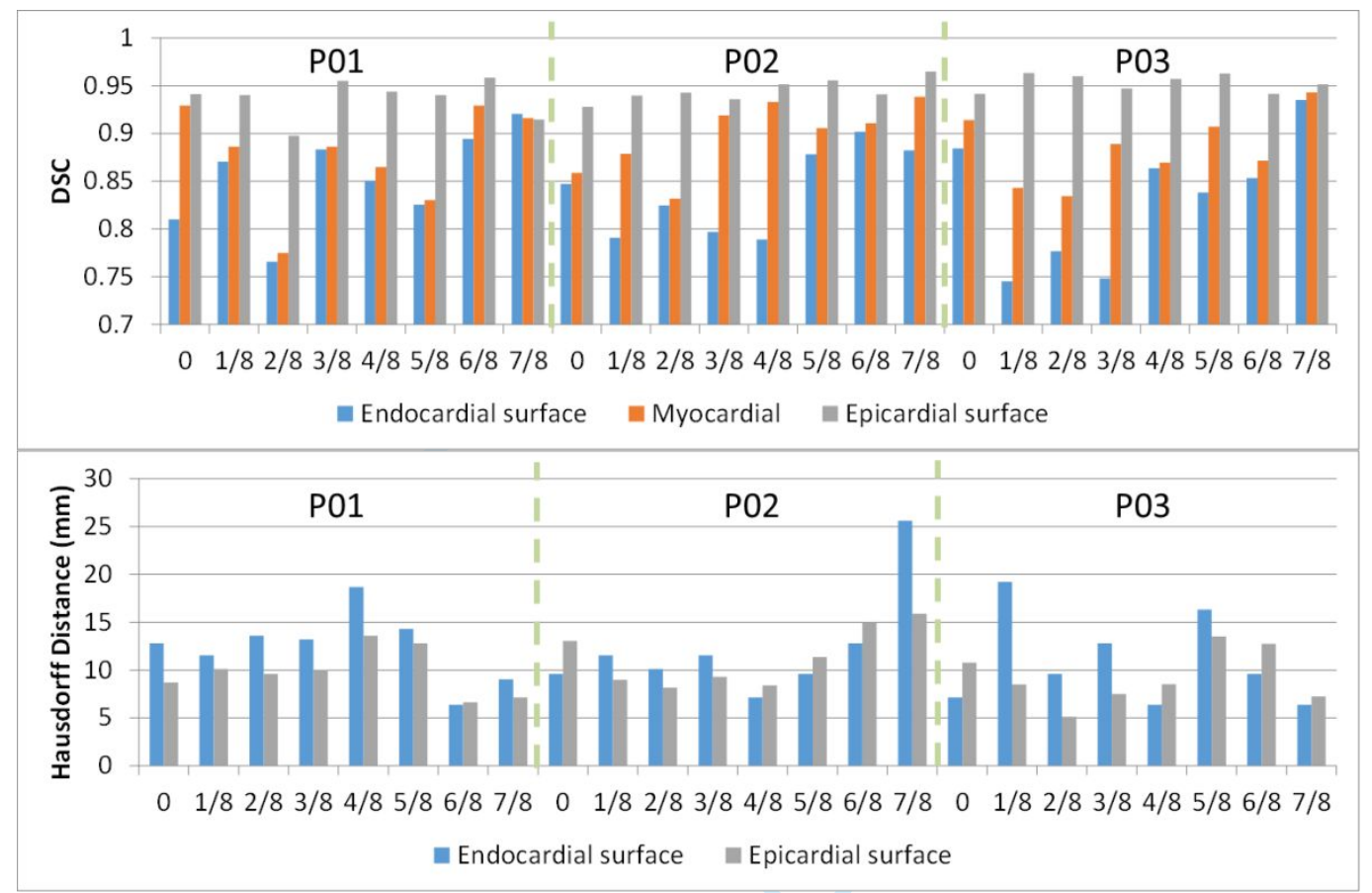

Fig. 12 DSC and Hausdorff distance of contours between two observers among 3 patients for each phase.

\section{DISCUSSION}

In this study, we proposed a novel machine-learning based method to segment LV and measure LV myocardium volume in gated MPS imaging. The average DSC metrics and Hausdorff distance of the contours delineated by our method are larger than 0.9 and less than $1 \mathrm{~cm}$, respectively. The results on abnormal patients are very similar with those on normal patients using our proposed method. The correlation coefficient of the LV myocardium volume between ground truth and results by the proposed method is $0.910 \pm 0.061$ with statistical significance, and the mean relative error of LV myocardium volume is $-1.09 \pm 3.66 \%$. These results strongly indicate the feasibility of the proposed method in accurately quantifing the changing LV myocardium volume 
during the cardiac cycle. It also demonstrates the great potential of learning-based segmentation method in gated MPS imaging for clinical use.

Segmentation of LV in MPS imaging studied in this paper is a critical step in clinical evaluations for quantifying multiple LV contractile functional indices. In this study, we validated the accuracy of LV myocardium volume measured by the proposed method with ground truth. An accurate LV myocardium volume measurement can predict adverse cardiovascular events and premature death based on a well-established model (24), and provides prognostic information beyond traditional cardiovascular disease risk factors (25). The endocardium contours segmented for all gating phases would also be tracked to calculate regional endocardial wall motion by computing the distance of the endocardial surface between end-diastole and end-systole. Thus, a segmentation method with high performance is essential to avoid introducing error from the beginning of MPS imaging practice.

Manual contours rely on observer's experience, and are reported to have substantial intraobserver and interobserver variability and less reproducibility (26). The manual contour from different observers may have systematic errors and random errors. Our learning-based method can mitigate random errors, but cannot correct systematic errors induced by the observers. In this study, we find that current contours for clinical use are represented as unsmooth curvature (see Fig. 2 and 3). The contours segmented by our method have better refinement, which is more physically plausible when considering real anatomical structures. Secondly, our method provides comparable results but spends significantly shorter time. With a trained model, it takes around 10 seconds to accurately delineate contours and measure volumes for all phases of MPS imaging on a NVIDIA TITAN XP GPU. Moreover, our method requires no manual input parameters, correction or intervention. Its speed and reproducibility allow it to be promising for clinical use.

Overestimation of LV myocardium volume for small heart and underestimation for large heart is commonly seen in current existing methods $(17,18)$, which may lead to systematic errors on patient groups with small or large hearts. In our result, such correlation between volume measurement error and volume size is not observed from Fig. 6. The linear correlation coefficient between volume error and volume size for all phases is - 
$0.222(\mathrm{P}=0.238)$ on average, which indicates very low correlation without statistical significance. Thus, our method is able to work equally well regardless of the size of LV myocardium volume.

We compared the EF calculated based on our segmentation results with that obtained from a commercial

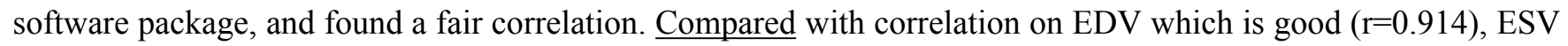
has larger discrepancy from commercial software $(r=0.818)$. Thus the differnce in the EF is mainly contributed from the difference in ESV. It may be explained by the different methodology used to determine ESV in the commercial software with various post-processing steps, which leads to larger discrpency in results from our method. Studies showed that the correlation of EFs between two commercial softwares using different methods could be around 0.800 (27). Thus, the commercial software results should be considered as a benchmark instead of a gold standard in this study.

Note that this study does not aim to demonstrate the absolute accuracy of the output contours of the proposed method by comparing with patients' true myocardial contours which are always unavailable. Instead, we showed the high correlation of the output contours with its training dataset, which is manual contour from one observer in this study. Such high correlation would still exist if the training contours are from another experienced observer since the method is not designed for a specific observer. Thus, if the training dataset is closer to the true patient contour (e.g. consensus contours by multiple observers), the result of our method would also be closer to the true contour. In other words, our method generates contours with similar quality as training contours, and the quality of training dataset directly determines the quality of the output results.

In this study, we proposed a novel method for MPS automatic segmentation, and demonstrated its feasibility with 32 normal patients and 24 abnormal patients. This training/testing dataset has intermediate number of patients with anatomical variations and pathology abnormalities. Future study would involve a comprehensive evaluation with a larger population of patients with diverse demographics and pathological abnormalities. Different testing and training datasets (including normal and abnormal cases) from different observers and institutes would be valuable to further evaluate the clinical utility of our method. Moreover, this study validated the proposed method by quantifying the shape similarity of contours. Small differences from ground truth are 
observed, and its potential clinical impact (e.g. on functional indices) needs to be understood. Thus, a further investigation in diagnostic accuracy of the proposed method in detection and localization of coronary artery disease would be of great interest for clinical use.

\section{CONCLUSION}

We proposed a learning-based method to automatically segment LV and measure LV myocardium volume in gated MPS imaging. This method would benefit the gated MPS imaging in providing high quality automatic quantification on multiple LV contractile functional indices without manual intervention. The proposed method was evaluated among 32 normal patients and 24 abnormal patients. The results demonstrate the feasibility of the proposed method in contouring with comparable accuracy as that based on physician experience.

\section{NEW KNOWLEDGE GAINED}

A learning-based method has been proposed to automatically segment LV and measure myocardium volume in gated MPS imaging. Results show that the proposed method has the feasibility in contouring with comparable accuracy as that based on physician experience.

\section{ACKNOWLEDGEMENTS}

This research is supported in part by the National Cancer Institute of the National Institutes of Health under award number R01CA215718 and Emory Winship Cancer Institute pilot grant. This research is also supported by the American Heart Association under Award number 17AIREA33700016.

\section{CONFLICT OF INTEREST}

None 


\section{REFERENCES}

(1) Dvorak RA, Brown RKJ, Corbett JR. Interpretation of SPECT/CT Myocardial Perfusion Images: Common Artifacts and Quality Control Techniques. RadioGraphics 2011;31:2041-57.

(2) Holder L, Lewis S, Abrames E, Wolin EA. Review of SPECT myocardial perfusion imaging. J Am Osteopath Coll Radiol 2016;5:5-13.

(3) Zhou W, Garcia EV. Nuclear Image-Guided Approaches for Cardiac Resynchronization Therapy (CRT). Current cardiology reports 2016;18:7.

(4) Garcia EV, Cooke CD, Van Train KF, Folks R, Peifer J, DePuey EG et al. Technical aspects of myocardial SPECT imaging with technetium-99m sestamibi. The American journal of cardiology 1990;66:23e-31e.

(5) Collom SJ, Case JA, Bateman TM. Electrocardiographically gated myocardial perfusion SPECT: Technical principles and quality control considerations. Journal of Nuclear Cardiology 1998;5:418-25.

(6) Paul AK, Nabi HA. Gated myocardial perfusion SPECT: basic principles, technical aspects, and clinical applications. Journal of nuclear medicine technology 2004;32:179-87; quiz 88-9.

(7) Shah PK, Pichler M, Berman DS, Singh BN, Swan HJ. Left ventricular ejection fraction determined by radionuclide ventriculography in early stages of first transmural myocardial infarction. Relation to shortterm prognosis. The American journal of cardiology 1980;45:542-6.

(8) Slomka P, Xu Y, Berman D, Germano G. Quantitative analysis of perfusion studies: strengths and pitfalls. Journal of nuclear cardiology : official publication of the American Society of Nuclear Cardiology 2012;19:338-46.

(9) Sharir T, Germano G, Kang X, Lewin HC, Miranda R, Cohen I et al. Prediction of myocardial infarction versus cardiac death by gated myocardial perfusion SPECT: risk stratification by the amount of stressinduced ischemia and the poststress ejection fraction. Journal of nuclear medicine : official publication, Society of Nuclear Medicine 2001;42:831-7.

(10) Sharir T, Berman DS, Waechter PB, Areeda J, Kavanagh PB, Gerlach J et al. Quantitative analysis of regional motion and thickening by gated myocardial perfusion SPECT: normal heterogeneity and criteria for abnormality. Journal of nuclear medicine : official publication, Society of Nuclear Medicine 2001;42:1630-8.

(11) Shaw LJ, Iskandrian AE. Prognostic value of gated myocardial perfusion SPECT. Journal of nuclear cardiology : official publication of the American Society of Nuclear Cardiology 2004;11:171-85.

(12) Friehling M, Chen J, Saba S, Bazaz R, Schwartzman D, Adelstein EC et al. A Prospective Pilot Study to Evaluate the Relationship Between Acute Change in Left Ventricular Synchrony After Cardiac Resynchronization Therapy and Patient Outcome Using a Single-Injection Gated SPECT Protocol. Circulation Cardiovascular imaging 2011;4:532-9.

(13) Faber TL, Cooke CD, Folks RD, Vansant JP, Nichols KJ, DePuey EG et al. Left ventricular function and perfusion from gated SPECT perfusion images: an integrated method. Journal of nuclear medicine : official publication, Society of Nuclear Medicine 1999;40:650-9.

(14) Xu Y, Kavanagh P, Fish M, Gerlach J, Ramesh A, Lemley M et al. Automated quality control for segmentation of myocardial perfusion SPECT. Journal of nuclear medicine : official publication, Society of Nuclear Medicine 2009;50:1418-26.

(15) Germano G, Kavanagh PB, Waechter P, Areeda J, Van Kriekinge S, Sharir T et al. A new algorithm for the quantitation of myocardial perfusion SPECT. I: technical principles and reproducibility. Journal of nuclear medicine : official publication, Society of Nuclear Medicine 2000;41:712-9.

(16) Germano G, Kiat H, Kavanagh PB, Moriel M, Mazzanti M, Su HT et al. Automatic quantification of ejection fraction from gated myocardial perfusion SPECT. Journal of nuclear medicine : official publication, Society of Nuclear Medicine 1995;36:2138-47.

(17) Eva P, Marcus C, John P, Olle P, Håkan A. Evaluation of left ventricular volumes and ejection fraction by automated gated myocardial SPECT versus cardiovascular magnetic resonance. Clinical Physiology and Functional Imaging 2005;25:135-41. 
(18) Soneson H, Ubachs JF, Ugander M, Arheden H, Heiberg E. An improved method for automatic segmentation of the left ventricle in myocardial perfusion SPECT. Journal of nuclear medicine : official publication, Society of Nuclear Medicine 2009;50:205-13.

(19) Bernard O, Lalande A, Zotti C, Cervenansky F, Yang X, Heng P et al. Deep Learning Techniques for Automatic MRI Cardiac Multi-structures Segmentation and Diagnosis: Is the Problem Solved? IEEE Transactions on Medical Imaging 2018:1-.

(20) Ngo TA, Lu Z, Carneiro G. Combining deep learning and level set for the automated segmentation of the left ventricle of the heart from cardiac cine magnetic resonance. Medical Image Analysis 2017;35:159-71.

(21) Betancur J, Rubeaux M, Fuchs TA, Otaki Y, Arnson Y, Slipczuk L et al. Automatic Valve Plane Localization in Myocardial Perfusion SPECT/CT by Machine Learning: Anatomic and Clinical Validation. Journal of nuclear medicine : official publication, Society of Nuclear Medicine 2017;58:961-7. (22) Milletari F, Navab N, Ahmadi S. V-Net: Fully Convolutional Neural Networks for Volumetric Medical Image Segmentation. 2016 Fourth International Conference on 3D Vision (3DV); 2016. p. 565-71.

(23) Varadhan R, Karangelis G, Krishnan K, Hui S. A framework for deformable image registration validation in radiotherapy clinical applications. Journal of applied clinical medical physics / American College of Medical Physics 2013; 14:4066-.

(24) Koren MJ, Devereux RB, Casale PN, Savage DD, Laragh JH. Relation of left ventricular mass and geometry to morbidity and mortality in uncomplicated essential hypertension. Annals of internal medicine 1991;114:345-52.

(25) Levy D, Garrison RJ, Savage DD, Kannel WB, Castelli WP. Left ventricular mass and incidence of coronary heart disease in an elderly cohort. The Framingham Heart Study. Annals of internal medicine 1989;110:101-7.

(26) Trobaugh GB, Wackers FJ, Sokole EB, DeRouen TA, Ritchie JL, Hamilton GW. Thallium-201 myocardial imaging: an interinstitutional study of observer variability. Journal of nuclear medicine : official publication, Society of Nuclear Medicine 1978;19:359-63.

(27) Nakajima K, Higuchi T, Taki J, Kawano M, Tonami N. Accuracy of ventricular volume and ejection fraction measured by gated myocardial SPECT: comparison of 4 software programs. Journal of nuclear medicine : official publication, Society of Nuclear Medicine 2001;42:1571-8. 


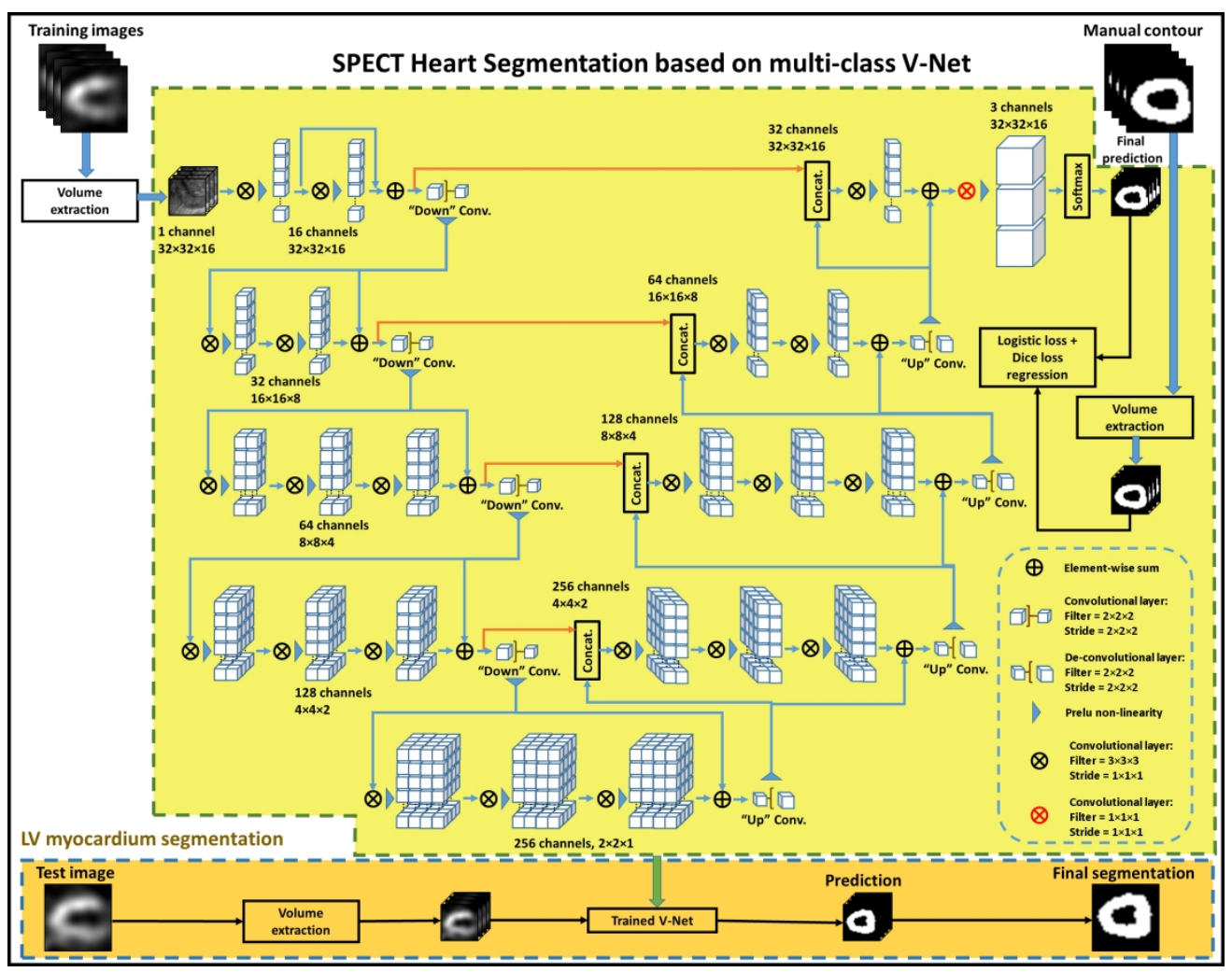

Fig. 1 Schematic flow chart of the proposed algorithm for LV segmentation. The upper part of this figure shows the training stage of our proposed method. The upper part also shows the V-Net architecture which has single channel volume input and 3 channels (background, region within endocardium, and region within epicardium) volume output. The lower part (brown) shows the segmentation stage. In segmentation stage, a new SPECT heart image is fed into the well-trained model to get the segmentation.

\section{$240 \times 188 \mathrm{~mm}(300 \times 300 \mathrm{DPI})$}



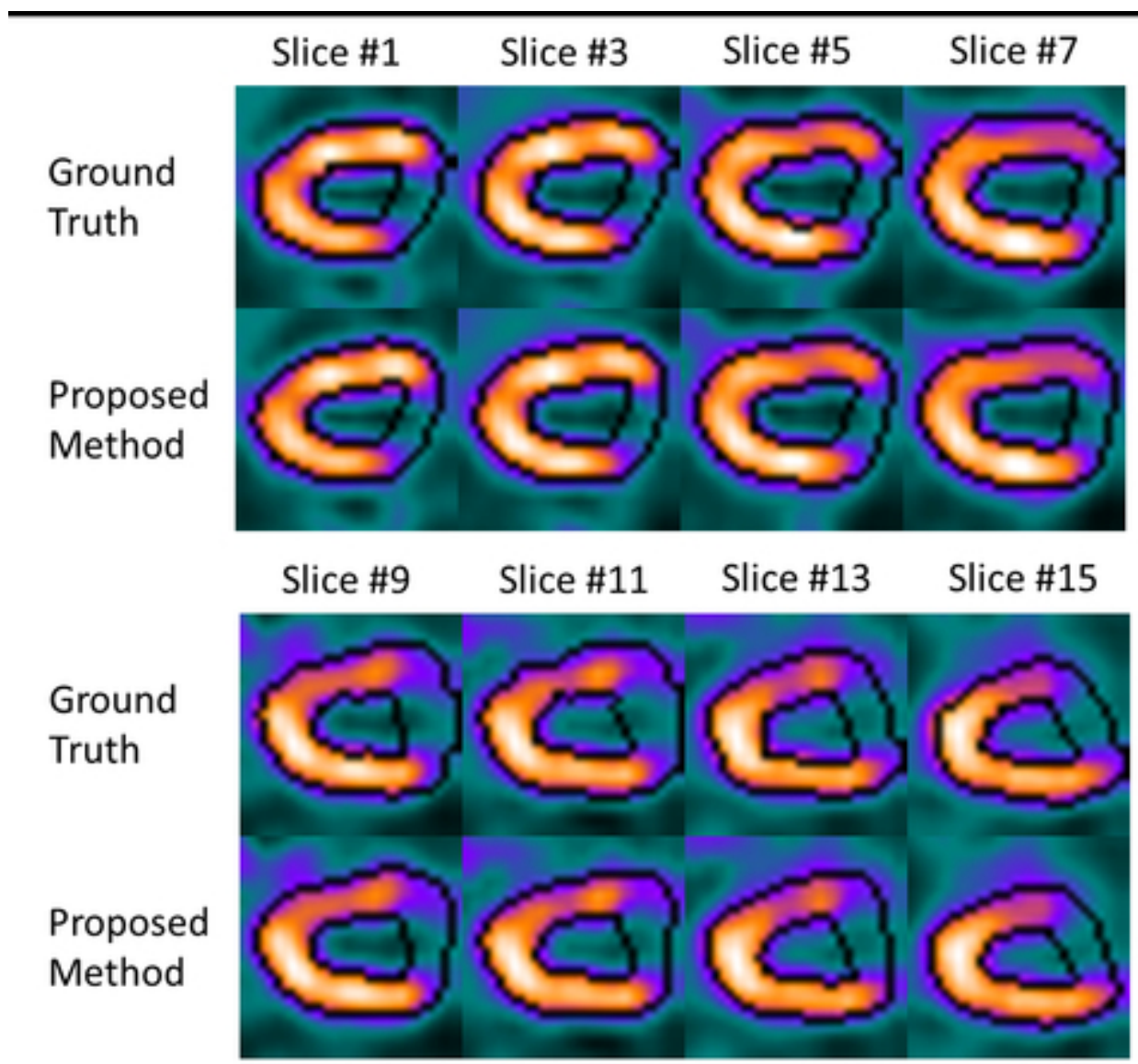

Fig. 2 The axial views of patient \#1 (normal) at different slices of gating phase 0 with segmentations of ground truth and proposed method. The black lines indicate the contours of endocardial and epicardial surface.

$40 \times 37 \mathrm{~mm}(300 \times 300$ DPI $)$ 

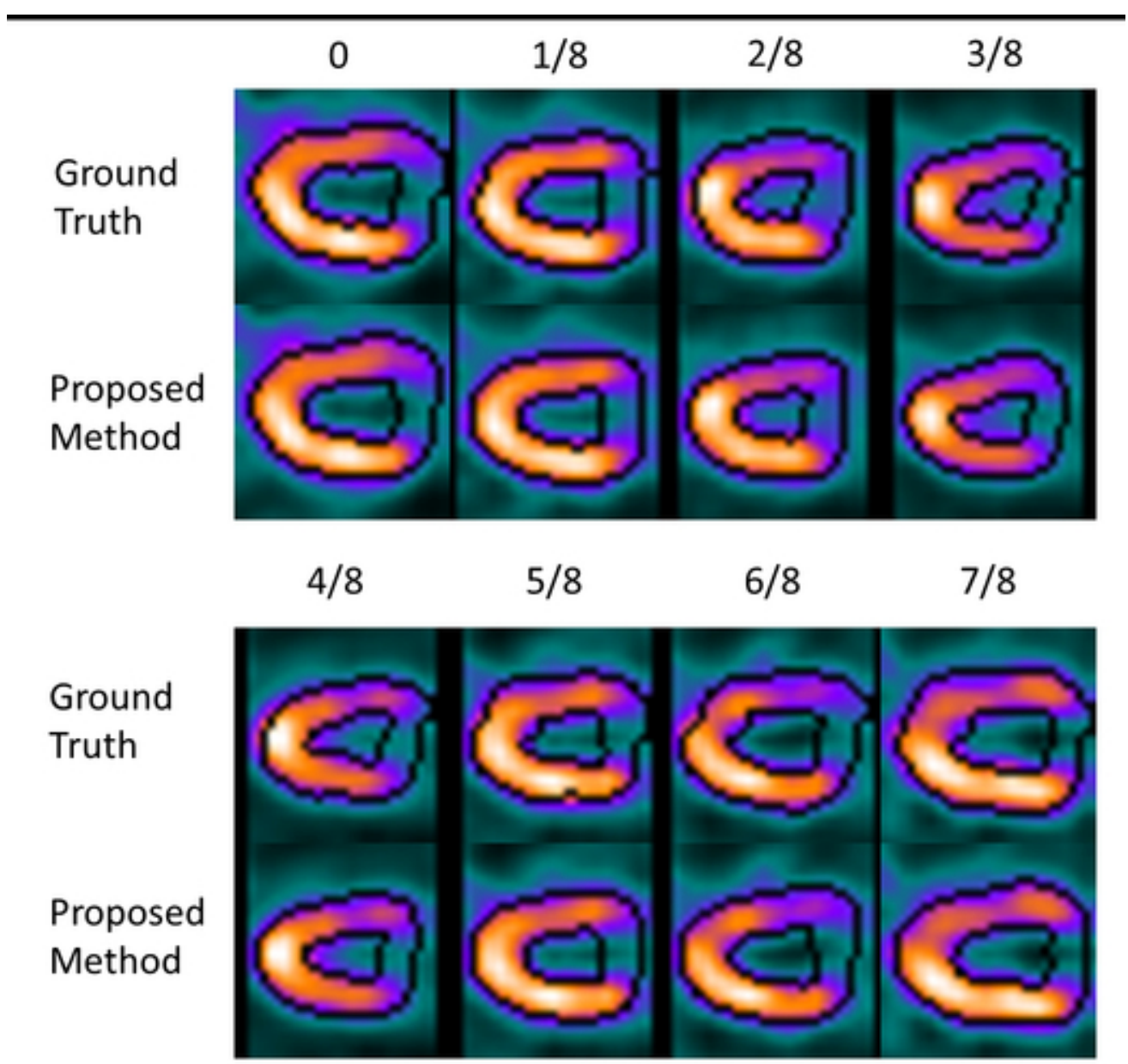

Fig. 3 The axial views of patient \#1 (normal) from phase 0 to $7 / 8$ at same slice with segmentations of ground truth and proposed method. The black lines indicate the contours of endocardial and epicardial surface.

$43 \times 41 \mathrm{~mm}(300 \times 300 \mathrm{DPI})$ 

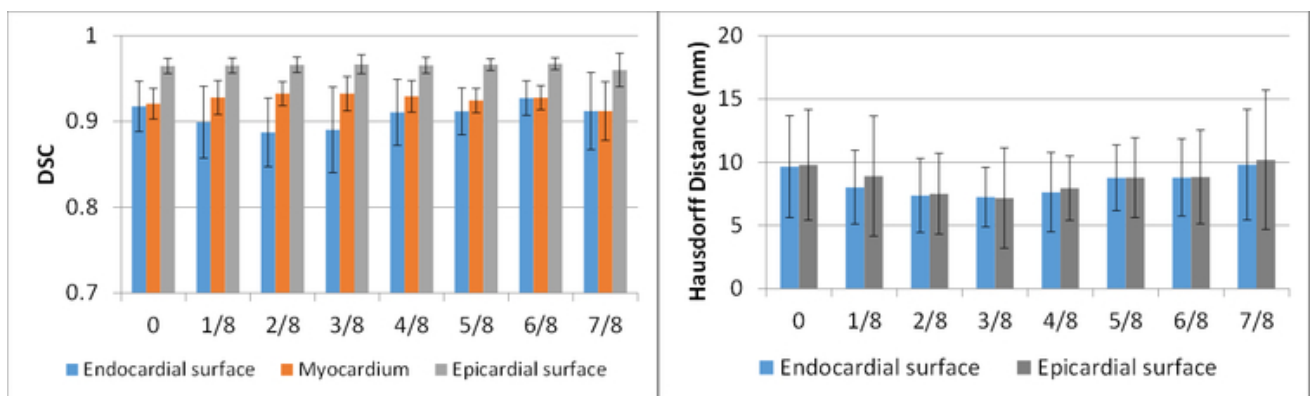

Fig. 4 Mean and STD of DSC and Hausdorff distance of contours between ground truth and proposed method among all 32 normal patients for each phase.

$63 \times 19 \mathrm{~mm}(300 \times 300 \mathrm{DPI})$ 


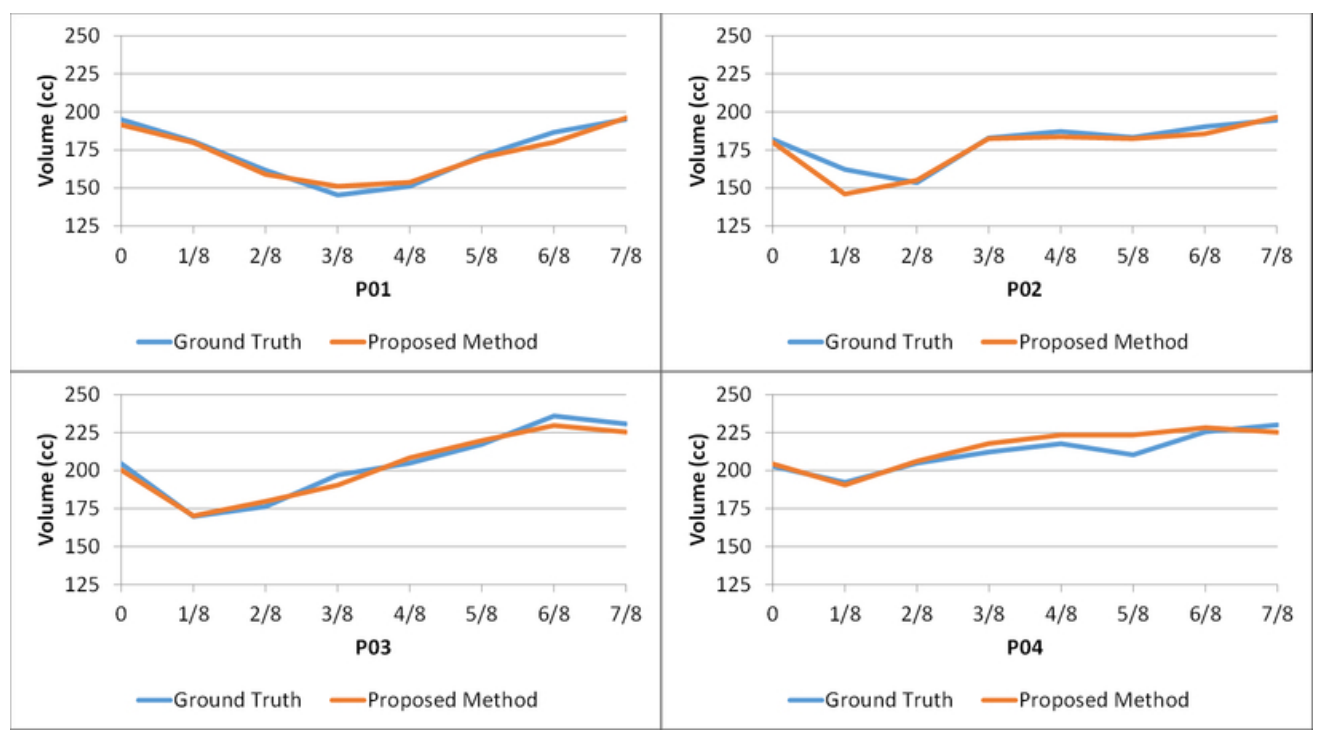

Fig. 5 LV myocardium volumes of ground truth and measured by ground truth at different phases of patient 01 to 04 (normal patients).

$69 \times 38 \mathrm{~mm}(300 \times 300 \mathrm{DPI})$ 

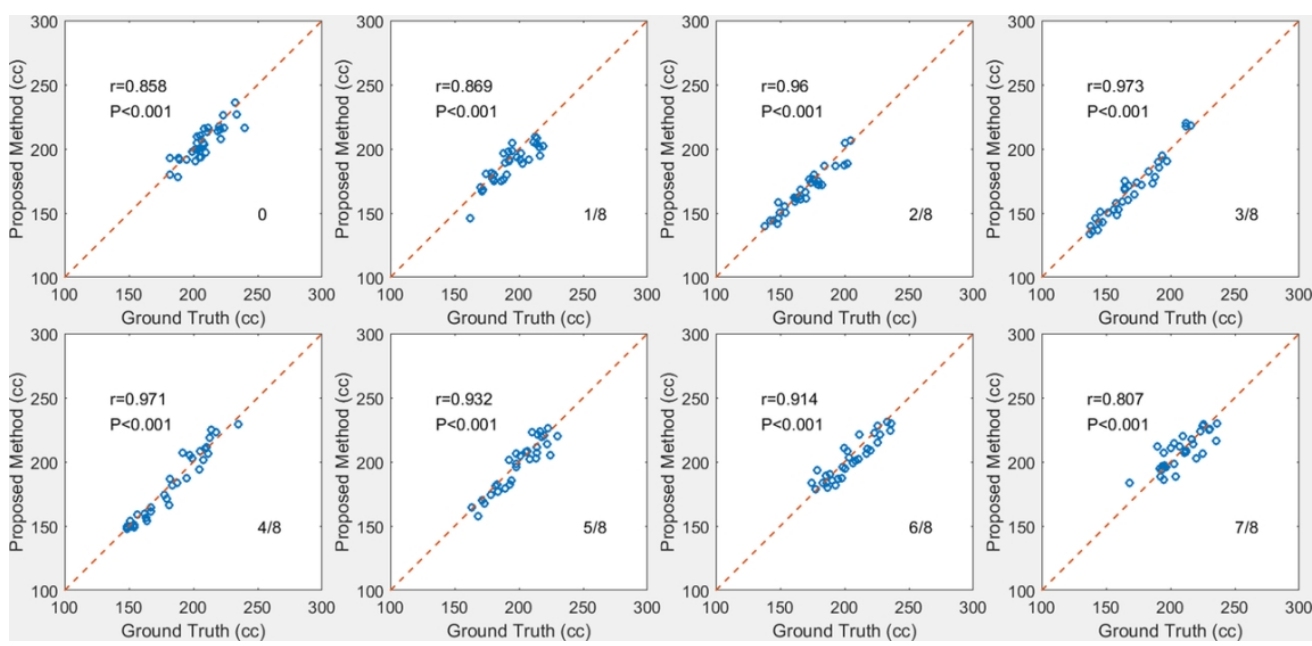

Fig. 6 Correlation analysis of LV myocardium volume between ground truth and proposed method at each gating phase among all 32 normal patients. Blue circle indicates measurement of each patient at that phase, and dashed red line is line of identity.

$108 \times 52 \mathrm{~mm}(300 \times 300 \mathrm{DPI})$ 


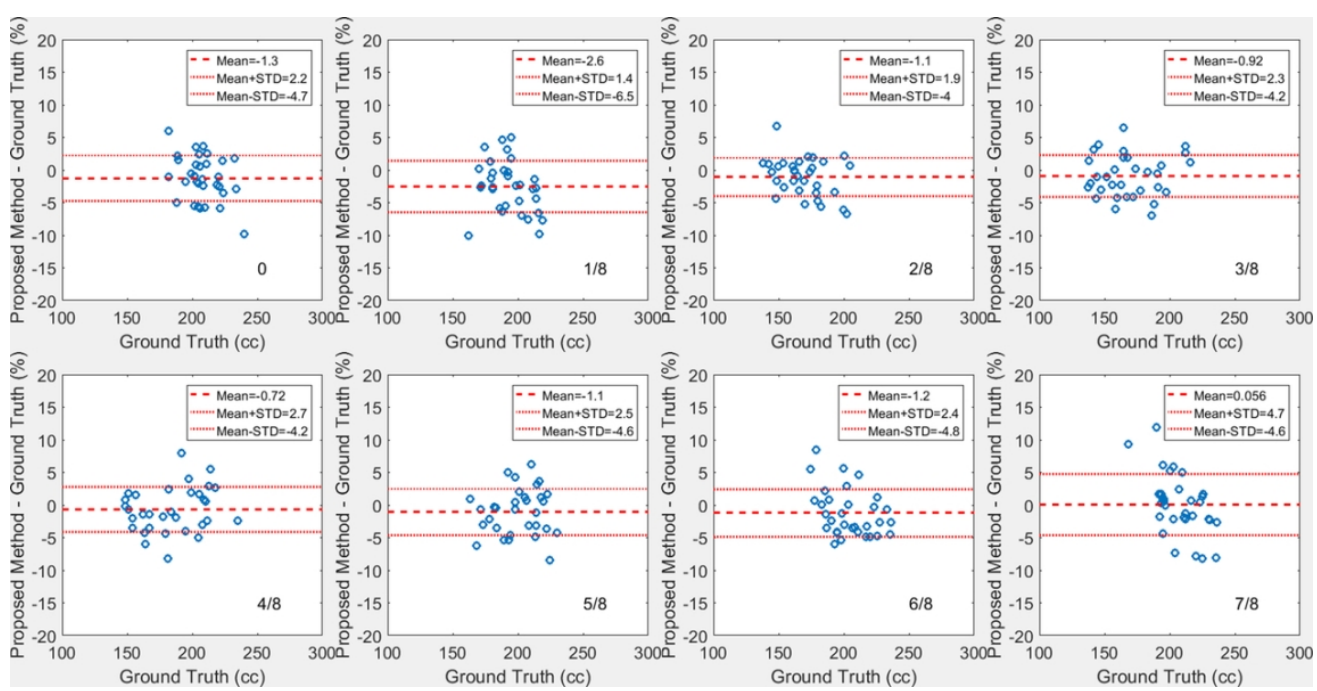

Fig. 7 Relative error of LV myocardium volume measured by the proposed method of each normal patient at each phase.

$107 \times 55 \mathrm{~mm}(300 \times 300 \mathrm{DPI})$ 

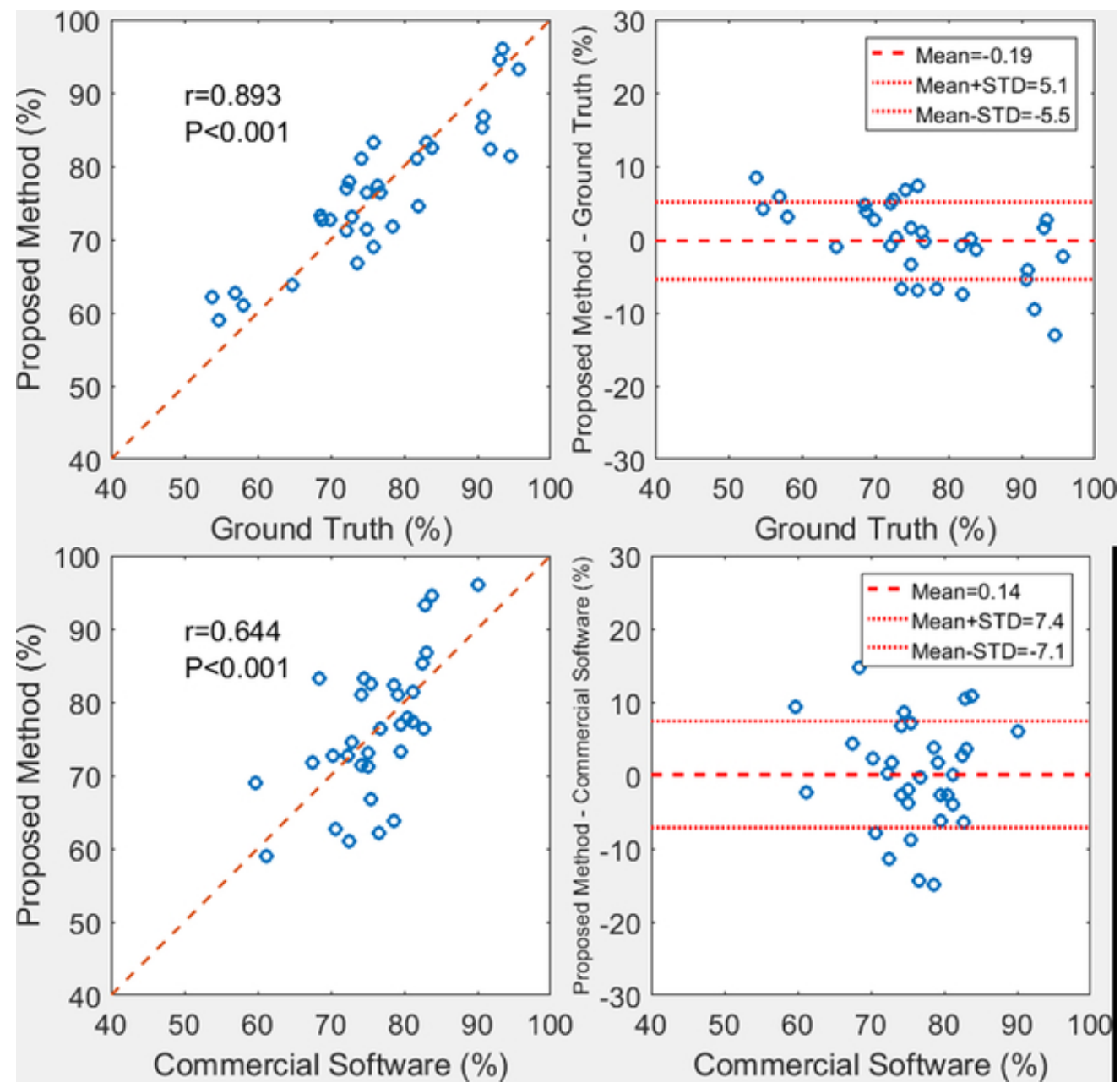

Left: Correlation analysis of EF between ground truth and proposed method (upper) and between commercial software and proposed method (bottom). Right: Difference of EF between ground truth and proposed method (upper) and between commercial software and proposed method (bottom).

$53 \times 52 \mathrm{~mm}(300 \times 300$ DPI $)$ 

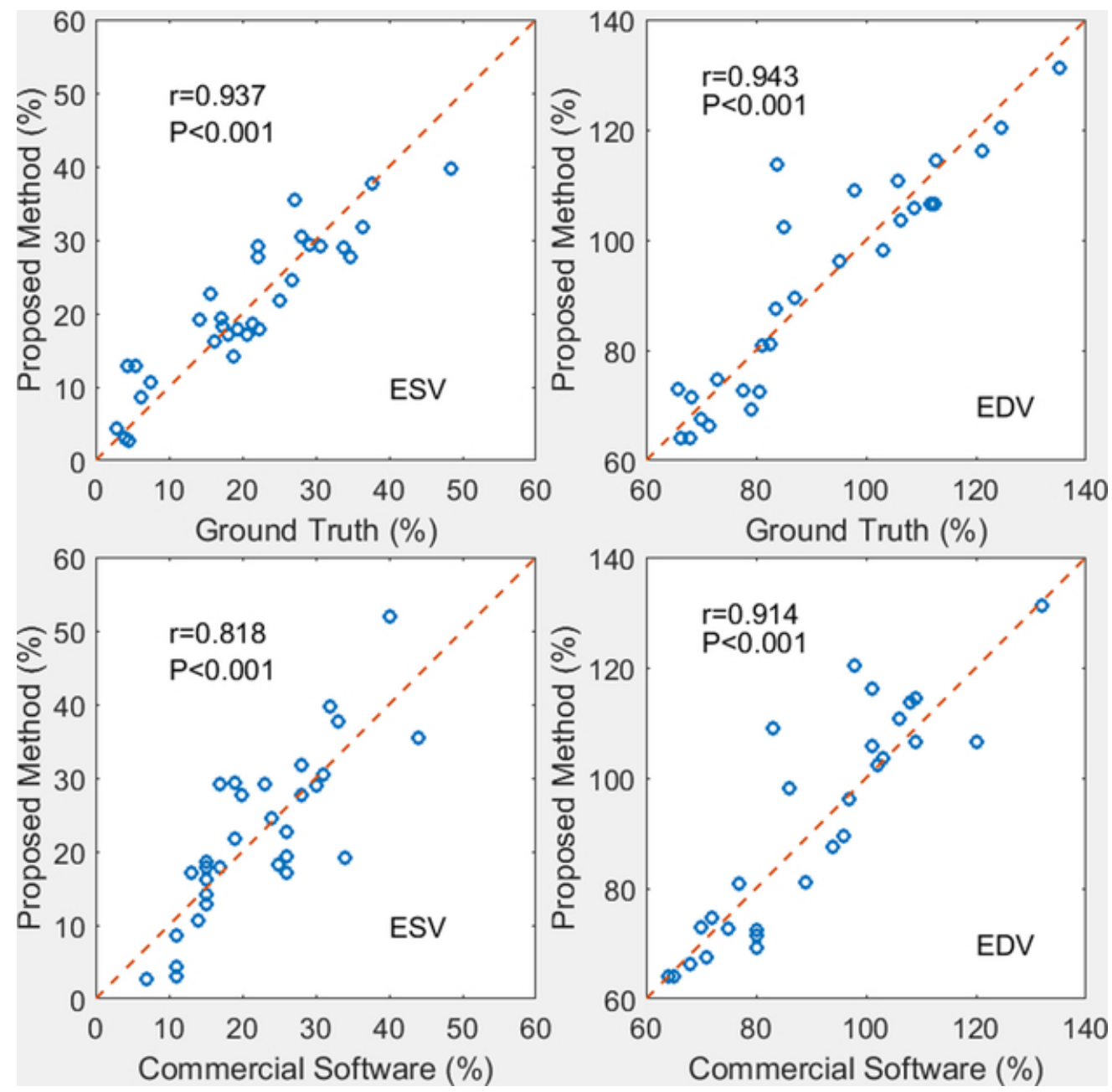

Correlation analysis of ESV (left) and EDV (right) between ground truth and proposed method (upper) and between commercial software and proposed method (bottom).

$53 \times 52 \mathrm{~mm}(300 \times 300 \mathrm{DPI})$ 


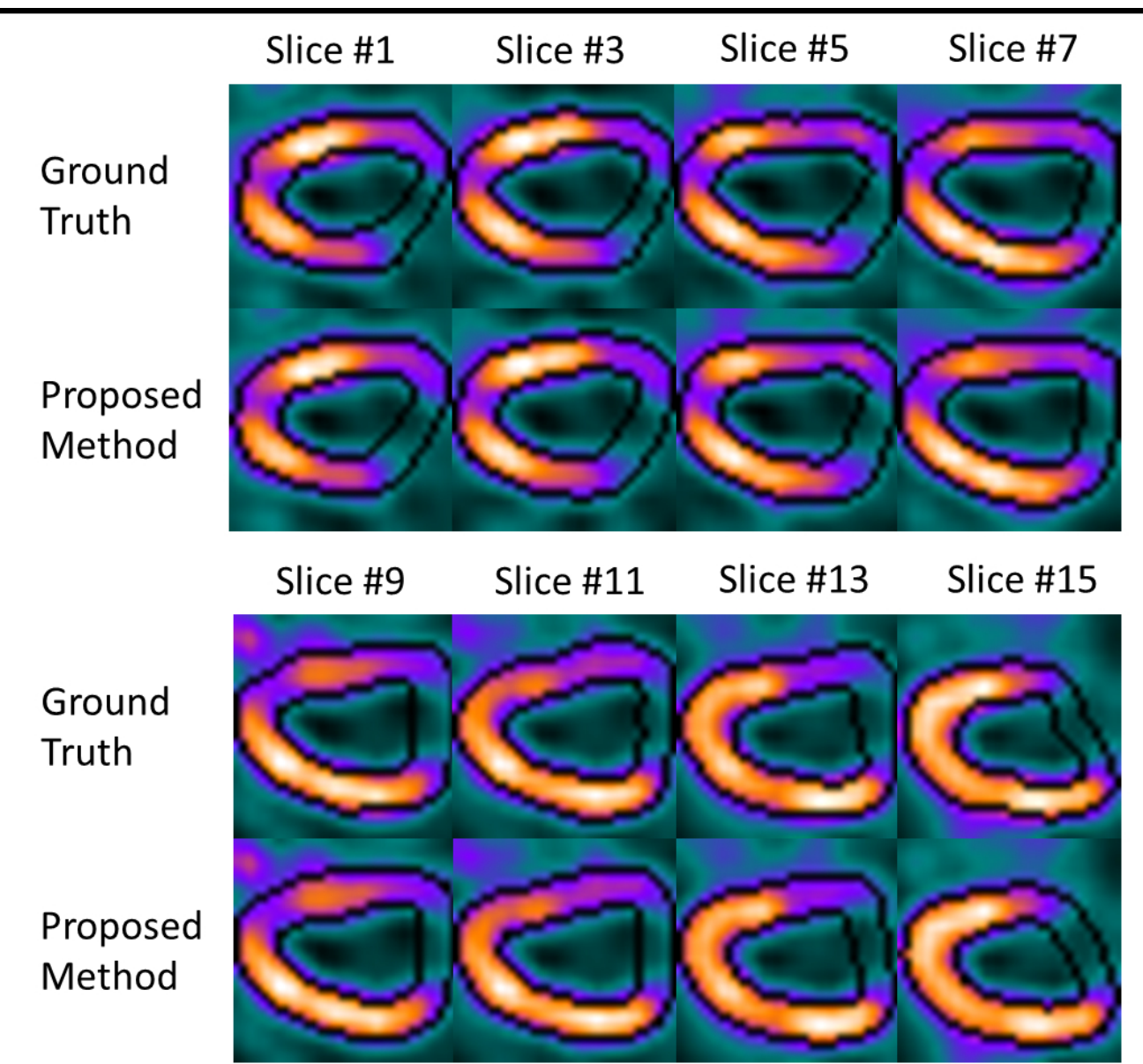

Fig. 10 The axial views of patient \#25 (abnormal) at different slices of gating phase 0 with segmentations of ground truth and proposed method. The black lines indicate the contours of endocardial and epicardial surface.

$86 \times 80 \mathrm{~mm}(300 \times 300$ DPI $)$ 


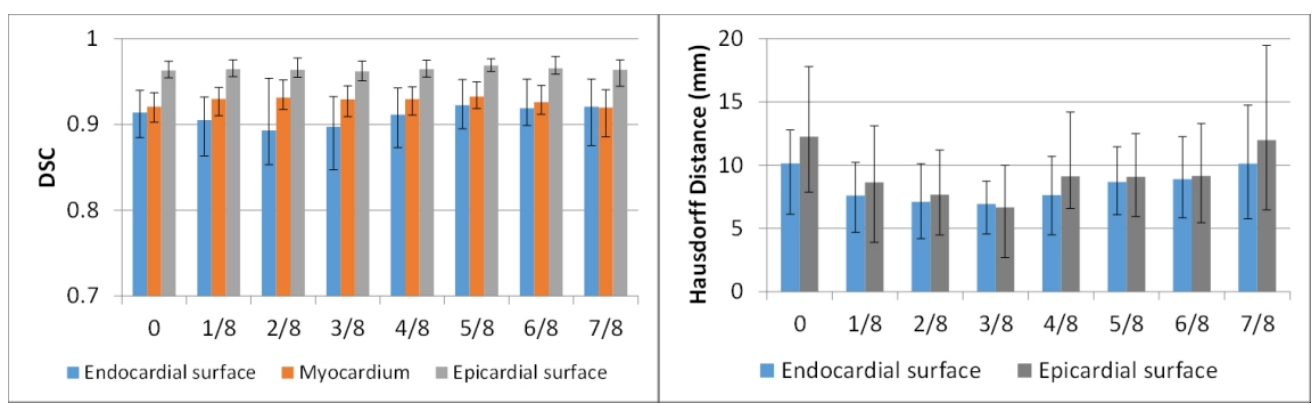

Fig. 11 Mean and STD of DSC and Hausdorff distance of contours between ground truth and proposed method among all 24 abnormal patients for each phase

$127 \times 38 \mathrm{~mm}(300 \times 300 \mathrm{DPI})$ 


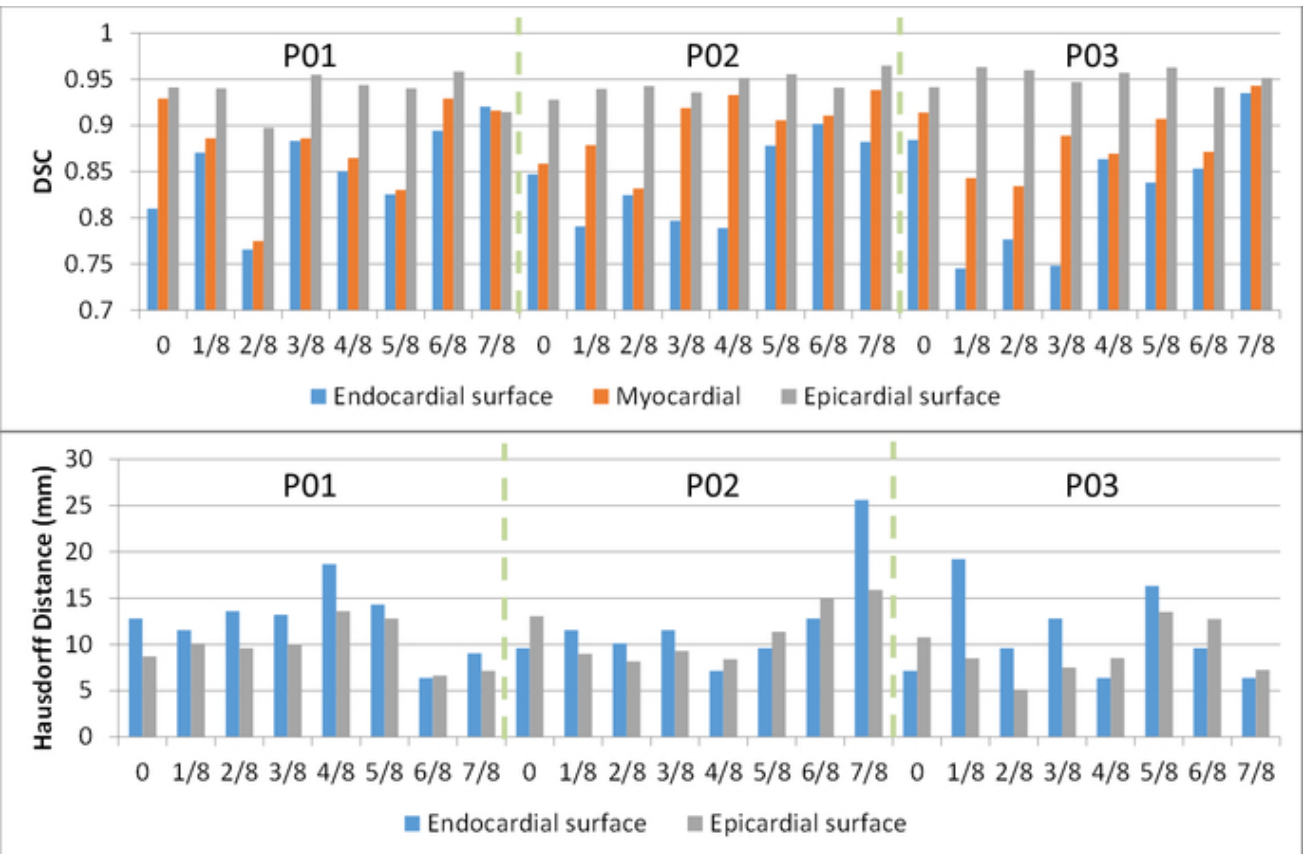

Fig. 12 DSC and Hausdorff distance of contours between two observers among 3 patients for each phase $58 \times 38 \mathrm{~mm}(300 \times 300$ DPI $)$ 
[General Response]: The authors wish to thank the reviewers and editor for the thorough review and constructive comments. Here we provide a point-by-point response to the reviewers' comments. In the revised manuscript, all the modifications that are based on reviewer comments are underlined.

\section{Referee \#1's Comments:}

Comments to the Author

The revised manuscript has clarified some of the issues raised during the previous round of review. However, some major concerns still remain.

1. It is stated that a leave-one-out procedure was used for training/testing the network, in which one patient was used for testing at each time while the rest 31 patients were used for training. During this process, were the hyper parameters of the network also varied for each test patient? For example, how was Imu determined?

[Response]: In order to fairly compare the performance of method on different patients, our hyper parameters of the network were fixed before we conducted the leave-one-out experiments. The batch size was set by 20 . The number of epochs was set to 180 . For the parameter $\mu$, we employed 4 -fold cross validation to evaluate the setting. It was shown that the performance is not sensitive when $\mu$ is between $[0.7,1.3]$, thus we set $\mu=1$.

2. For the method to be useful, abnormal studies need to be included in the evaluation.

[Response]: We agree with the reviewer. In the revised paper, we included 24 abnormal patients to test the proposed segmentation method. The 24 abnormal patients were diagnosed by SPECT MPI studies to have mild to severe myocardial ischemia (mild: 11; moderate: 11; severe: 2 ). These 24 abnormal patients were combined with 32 normal patients to train the deep-learning model and were evaluated with a leave-one-out validation strategy. Their results were reported by similar evaluation methods as we did for normal patients. The DSC of results on abnormal patients is $0.910 \pm 0.037,0.927 \pm 0.018$ and $0.965 \pm 0.011$ on endocardium surface, myocardium and epicardium surface, respectively. The Hausdorff Distance is $8.384 \pm 3.240 \mathrm{~mm}$ and $9.310 \pm 5.034 \mathrm{~mm}$ on endocardium surface and epicardium surface, respectively. Overall, these results on abnormal patients are very similar with those of normal patients, with mean DSC larger than 0.9 and Hausdorff distance less than $1 \mathrm{~cm}$. We included these results in our revised paper.

"In addition, 24 patients (mean \pm STD age: 57 $\pm 10,17$ males, 7 females) diagnosed with myocardial ischemia ranging from mild, moderate to severe extents were also included to further test the proposed segmentation method with leave-one-out strategy."

“Fig. 10 demonstrates a side-by-side comparison between our results and the clinical ground truth at different slices of gating phase 0 from patient \#25 (diagnosed with moderate ischemia) as an example. In this case, the LV myocardium volume measured by the proposed method was $212.0 \mathrm{cc}$, overestimated $1.49 \%$ from ground truth $208.9 \mathrm{cc}$. The mean and standard deviation (STD) of DSC of endocardial and epicardial surface, and myocardium, and Hausdorff distance of endocardial and epicardial surface among all 24 abnormal patients are plotted in Fig. 11 for each phase and summarized in Table 2. Overall, the results on abnormal patients are very similar with those of normal patients, with mean DSC larger than 0.9 and Hausdorff distance less than $1 \mathrm{~cm}$. The correlation coefficient of the LV myocardium volume 
between ground truth and our results is $0.939 \pm 0.103(P<0.001)$, and the mean relative error of LV myocardium volume is $-0.567 \pm 3.47 \%$.

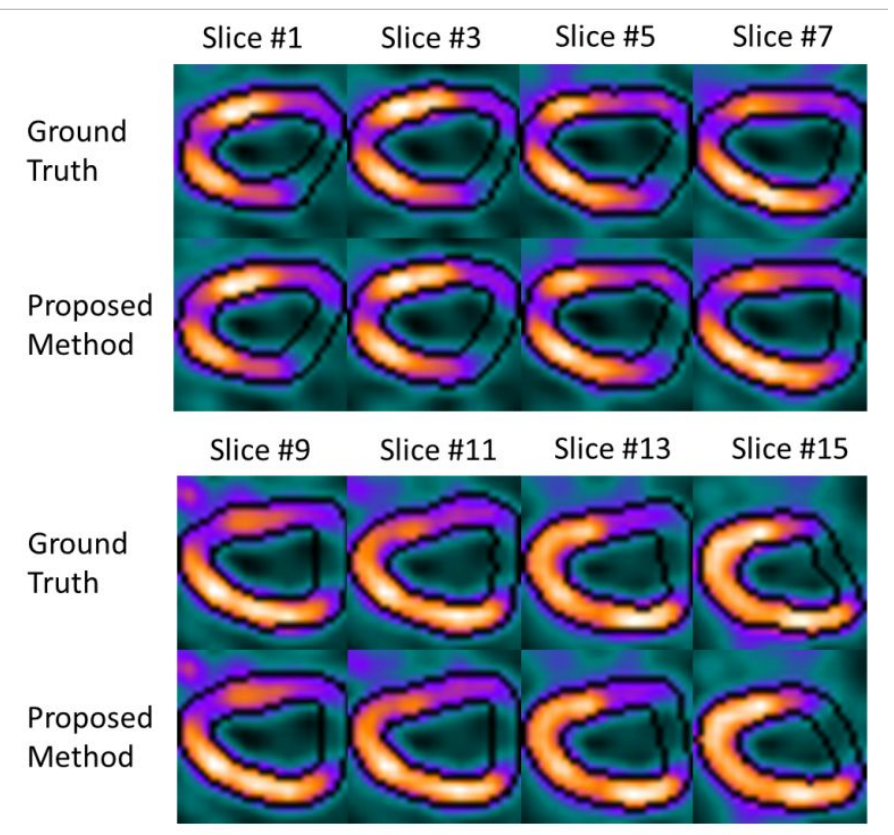

Fig. 10 The axial views of patient \#25 (abnormal) at different slices of gating phase 0 with segmentations of ground truth and proposed method. The black lines indicate the contours of endocardial and epicardial surface.
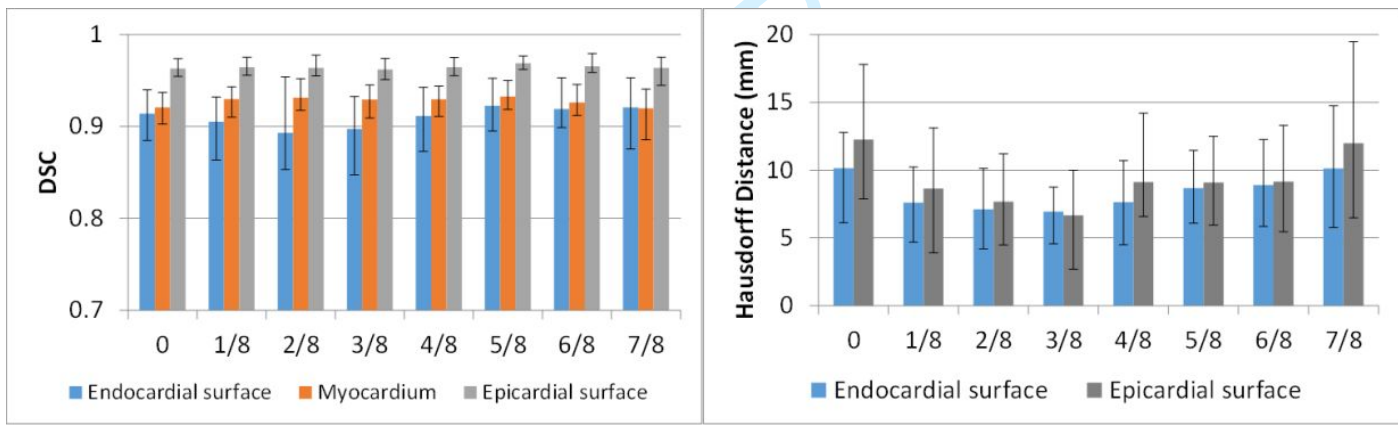

Fig. 11 Mean and STD of DSC and Hausdorff distance of contours between ground truth and the proposed method among all 24 abnormal patients for each phase.

Table 2. Mean \pm STD of DSC and Hausdorff distance among all 24 abnormal patients.

\begin{tabular}{cccc}
\hline \hline Metrics & Endocardium & Myocardium & Epicardium \\
\hline DSC & $0.910 \pm 0.037$ & $0.927 \pm 0.018$ & $0.965 \pm 0.011$ \\
Hausdorff Distance $(\mathrm{mm})$ & $8.384 \pm 3.240$ & $\mathrm{~N} / \mathrm{A}$ & $9.310 \pm 5.034$ \\
\hline \hline
\end{tabular}

3. P.5, line 12 , how was the $3 D$ region $(32 \times 32 \times 16)$ "automatically" cropped? 
[Response]: Each original SPECT heart image includes a large area of background as shown in the following figure. The intensity in active area is around 200, which is higher than the background region (30-40). Thus, we used a threshold (100) to first get rid of background and calculated the centroid of the active heart region, then we centrally crop the image to a region of $32 \times 32 \times 16$ voxels, which is big enough to cover the active heart region.

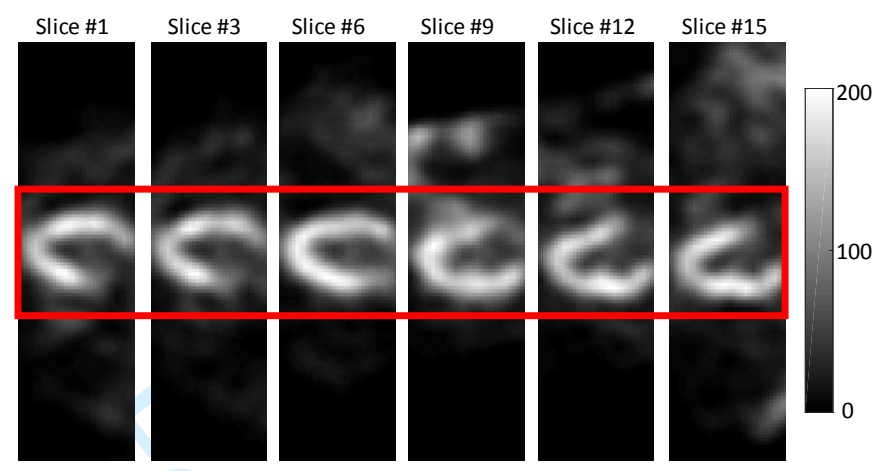

Fig. R1. Example of SPECT heart images. From left to right it shows different slices of SPECT heart image. The display window is $[0,200]$.

We added this comment in our revised paper:

"The original SPECT images were first automatically cropped into $32 \times 32 \times 16$ voxels to reduce background region: a threshold was used to get rid of background and the centroid of the active heart region was then calculated, based on which a $32 \times 32 \times 16$ voxel region was cropped to cover the active heart region."

4. P.6, line 16, "To address this issue, we additionally incorporated the logistic loss with Dice loss in the final stages as the final objective function." Is there any theoretical justification that your approach can address the local minima issue?

[Response]: In segmentation task, the local minima issue often exists when the target region is much smaller than the region outside the target region. In SPECT heart segmentation, the region within endocardium is much smaller than the region outside the endocardium. If we use equal loss weighting strategy in gradient descent optimization, e.g., the same loss weight for the segmenting region within endocardium and segmenting region outside the endocardium, this strategy would cause the learning process to get trapped in local minima of the loss function whose predictions are strongly biased towards the larger one (the region outside the endocardium). In order to solve this potential, we aim to use a more suitable weighting strategy. In fact, in our Adam gradient descent optimization when training the network, the weight of loss is obtained by the gradient of our loss function. Milletari et al. demonstrated the gradient of Dice loss can assign a balance weight for different regions in volume based segmentation ${ }^{1}$. We used the same weighting strategy as recommended in Milletari's study. For the details, please see the Section 3 of this paper for more details ${ }^{1}$.

5. It is stated that the coefficient Imu was empirically determined to achieve the best performance. Was this best performance determined from test cases? If so, wouldn't this lead to a favorable bias?

[Response]: Thanks for the comment. The parameter $\mu$ was not determined from test cases. It was fixed before our leave-one-out experiments. Thus, it would not lead to a favorable bias. We modified the 
parameter $\mu$ setting in our revised manuscript as follows "We employed 4-fold cross validation to evaluate its setting. It is shown that the performance is not sensitive when $\mu$ is between $[0.7,1.3]$, thus we set $\underline{\mu} \equiv \underline{1 . "}$

6. The comparison results show only a fair correlation $(r=0.644)$ in EF between the proposal approach and the commercial tool. This raises a question about whether the proposed approach is more accurate than the commercial tool.

[Response]: On our segmentation results, we chose the maximum and minimum volumes within endocardial surface among all phases as EDV and ESV. However, commercial software determines EDV and ESV with sophisticated post-processing steps in modeling the whole systolic and diastolic period. Such difference in determining EDV and ESV leads to the discrepancy of EF between our results and commercial software. Implementing a similar post-processing for EDV and ESV determination modeling as commercial software is out of the scope of this study. We also discussed this in our discussion section.

7. The results in Fig. 5 show notable variability between the two observers on three cases. How will this affect the reliability of the evaluation results?

[Response]: This study does not aim to demonstrate the absolute accuracy of the output contours of the proposed method by comparing with patients' true myocardial contours which are always unavailable. Instead, we trained our model based on one observer's contour, and evaluated our results using the contour from the same observer, and we showed the high correlation of the output contours with its training dataset. Such high correlation would still exist if the training contours are from another experienced observer since the method is not designed for a specific observer. Interobserver variability is not involved into the evaluation process, since it is fair only when the contours from the same observer or same group of observers are to be used for both training and evaluation. The manual contour from different observers may have systematic errors and random errors. Our learning-based method can mitigate random errors, but cannot correct systematic errors induced by the observers. We also discussed this in our discussion section.

\section{Referee \#2's Comments:}

Comments to the Author

The authors have made many changes to their manuscript that certainly clarify a number of things. However, I do have some questions remaining, in particular regarding the description of the method.

One major limitation of this work is that only healthy patients are included, and the data set is quite small (only 32 patients). That the myocardium can be efficiently segmented in these high-contrast, small images of a reasonably standardized group is not very surprising. It would be much more interesting to find out how well this works in patients with pathology.

[Response]: We agree with the reviewer. As we respond to the first reviewer, in the revised paper, we included 24 abnormal patients to test the proposed method with a leave-one-out validation strategy. Their results were reported by similar evaluation method as we did for normal patients. The DSC of results on abnormal patients are $0.910 \pm 0.037,0.927 \pm 0.018$ and $0.965 \pm 0.011$ on endocardium surface, myocardium and epicardium surface, respectively. The Hausdorff Distance is $8.384 \pm 3.240 \mathrm{~mm}$ and $9.310 \pm 5.034 \mathrm{~mm}$ on endocardium surface and epicardium surface, respectively. Overall, these results on abnormal patients are very similar with those of normal patients, with mean DSC larger than 0.9 and Hausdorff distance less than $1 \mathrm{~cm}$. We included these results in our revised paper. 


\section{Major comments}

Although it is now much clearer that you are trying to segment the myocardium, it is still unclear what the segmentation target of the $V$-Net is. Is this a binary mask where voxels between the endocardial and epicardial contour have been set to 1 ? Or do you have multiple masks? I see in Fig. 1 that there are 4 output channels, but I don't understand from the text where these 4 channels/classes are coming from. Are you segmenting the areas within the endocardial and epicardial contours separately, is that it? Shouldn't this then lead to 3 output channels?

On a related note, the neural network performs 'multi-label' segmentation according to the authors. As far as I know, this means that multiple labels can be predicted per voxel. Yet a softmax layer is applied to the four probability channels, which suggests that you're actually performing multi-class instead of multi-label classification. Please see an explanation of this distinction at e.g. https://scikitlearn.org/stable/modules/multiclass.html

[Response]: We agree with the reviewer. As the reviewer commented, we have 3 output channels. We corrected the related text and figure in our revised manuscript. The segmentation targets of the V-Net are the region within endocardium surface, the region within epicardium surface, and the region outside epicardium surface (background). The myocardium region is obtained by the subtraction of the region within endocardium surface from the region within epicardium. We have three masks, and set these masks as multi-channel outputs for supervision, e.g., we set mask as 1 for the region within endocardium, and 0 for other regions. 


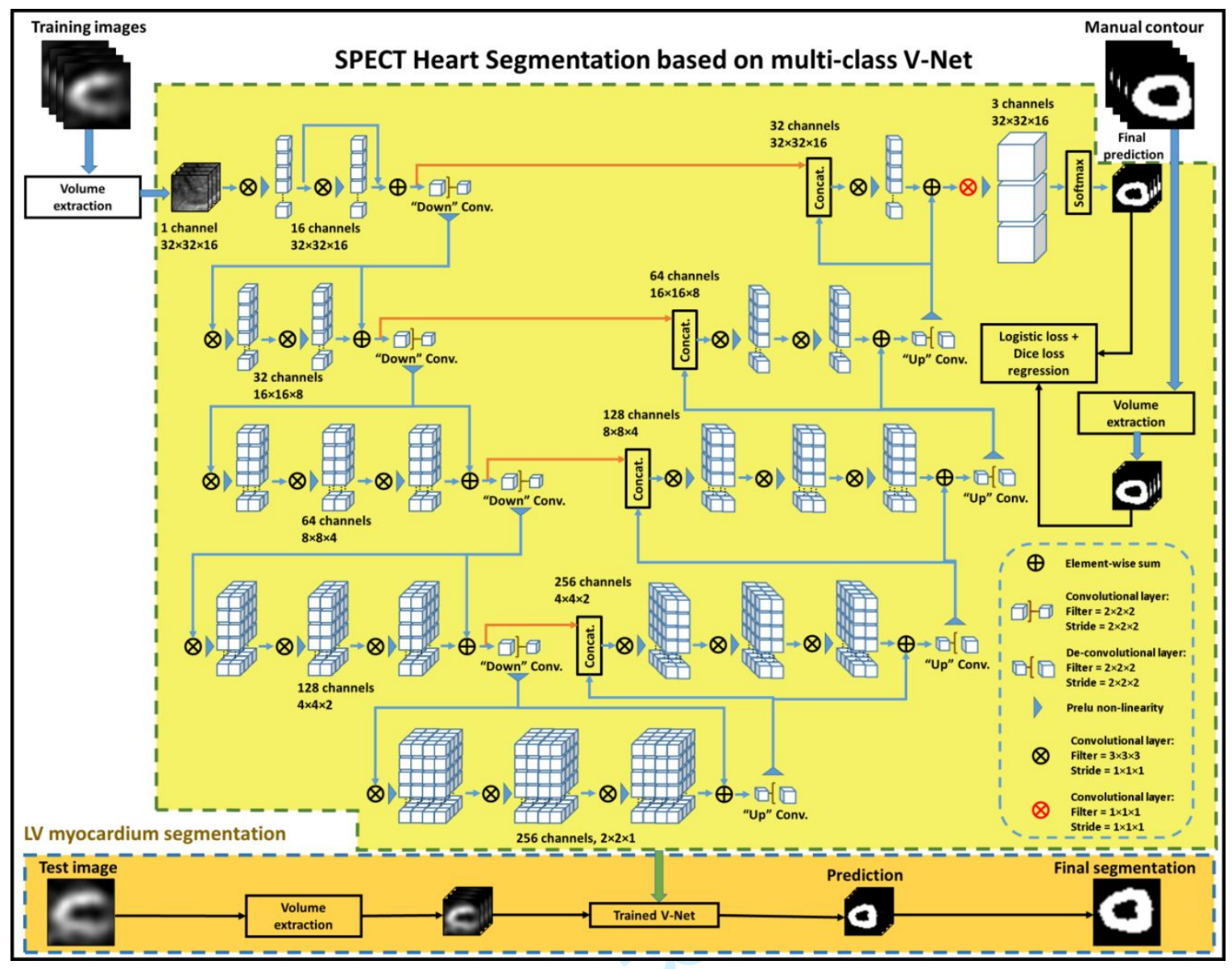

Fig. 1 Schematic flow chart of the proposed algorithm for LV segmentation. The upper part of this figure shows the training stage of our proposed method. The upper part also show the V-Net architecture which has single channel volume input and 3 channels (background, region within endocardium, and region within epicardium) volume output. The lower part (yellow) shows the segmentation stage. In segmentation stage, a new SPECT heart image is fed into the well-trained model to get the segmentation.

We appreciate reviewer's suggestion. According to the explanation of "multi-label" and "multi-class" classification at https://scikit-learn.org/stable/modules/multiclass.html, we agree with reviewer's comment and we changed "multi-label" into "multi-class" in our revised manuscript.

I don't think the Dice score in Eq. (2) is differentiable. Is this really what you use as a loss function?

[Response]: Dice loss has been used for many segmentation methods. Milletari et al. used Dice loss in their V-Net-based segmentation method. ${ }^{1}$ In their study, they reported that Dice loss can be differentiated yielding the gradient computed with respect to each voxel of the prediction. We agree with reviewer that a Dice score as a number is not differentiable. However, the Dice loss is not a score in our task. Our model is iteratively trained by Adam gradient descent optimizer. For each iteration, the training samples were fed into the model to generate the multi-channel output mask. The Dice loss is used to evaluate the similarity or overlap between generated mask with ground truth mask (the region within manual contour). Thus, Dice is a monotonically increasing function of overlap between generated mask and manual mask, and it can be used for iteratively gradient descent method. 
There is some mention of 'automatic cropping' of the volumes to reduce the size to $32 \times 32 \times 16$ voxels, yet this step remains unclear. What is the voxel size after this cropping?

[Response]: Each original SPECT heart image includes a large area of background as shown the following figure. The intensity in active area is around 200, which is higher than the background region (30-40). Thus, we used a threshold (100) to first get rid of background and calculated the centroid of the active heart region, then we centrally crop the image to a region with $32 \times 32 \times 16$ voxels, which is big enough to cover the active heart region. The voxel size (resolution) is not changed from original size after automatic cropping.

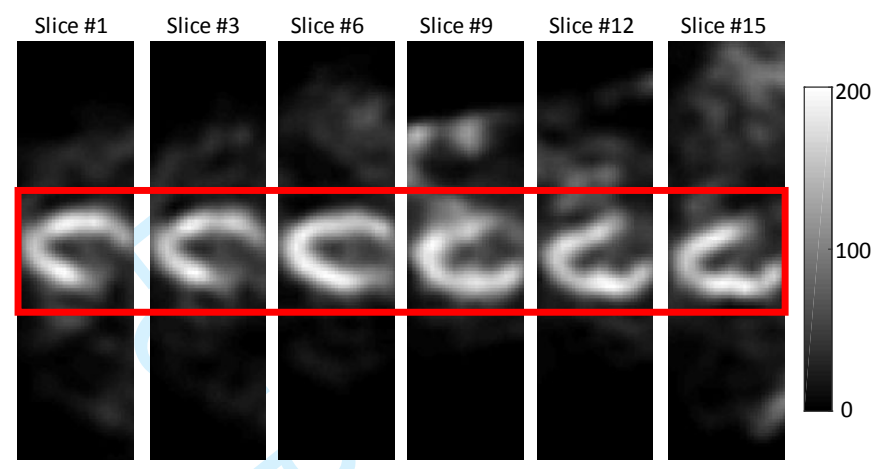

Fig. R1. Example of SPECT heart images. From left to right it shows different slices of SPECT heart image. The display window is $[0,200]$.

We modified this in our revised manuscript as "The original SPECT images were first automatically cropped into $32 \times 32 \times 16$ voxels to reduce background region."

Captions should really be more descriptive. Fig. 1 is a highly complicated figure, with one short line caption.

[Response]: We modified the caption of Fig.1 as follows "Schematic flow chart of the proposed algorithm for LV segmentation. The upper part of this figure shows the training stage of our proposed method. The upper part also show the V-Net architecture which has single channel volume input and 3 channels (background, region within endocardium, and region within epicardium) volume output. The lower part (yellow) shows the segmentation stage. In segmentation stage, a new SPECT heart image is fed into the well-trained model to get the segmentation."

\section{Minor comments}

The sentence 'The 3D multi-label V-Net architecture was introduced to enable ..' is not correct, the authors don't introduce V-Net, Milletari et al. (22) did.

[Response]: We corrected this statement.

I don't think you use the word 'modest' correctly. Maybe you mean 'intermediate'.

[Response]: We corrected this word.

Black is probably not the best color to show segmentations in Fig. 2/3. 


\section{Associate Editor \\ Comments to the Author: \\ Major issues still remain and must be definitively resolved in the revision.}

As reviewers mention 32 patients is a very small set. Further, authors must add some abnormal cases for this work to have some meaningful clinical validation.

[Response]: As we respond to the reviewers, in the revised paper, we included 24 abnormal patients to further test the proposed method with leave-one-out strategy. Their results were reported by similar evaluation method as we did for normal patients. Overall, these results on abnormal patients are very similar with those of normal patients, with mean DSC larger than 0.9 and Hausdorff distance less than $1 \mathrm{~cm}$. We included these results in our revised paper.

The title, the abstract, and text should prominently mention that this is a feasibility study only. The accuracy cannot be meaningfully evaluated in such small dataset. The manuscript needs to be thoroughly revised to reflect this.

[Response]: We revised the title as "A Learning-based Automatic Segmentation and Quantification Method on Left Ventricle in Gated Myocardial Perfusion SPECT Imaging: A Feasibility Study", and also revised the abstract and text to reflect this point.

\section{Reference}

1. Milletari F, Navab N, Ahmadi S. V-Net: Fully Convolutional Neural Networks for Volumetric Medical Image Segmentation. Paper presented at: 2016 Fourth International Conference on 3D Vision (3DV); 25-28 Oct. 2016, 2016. 